Open Access

\title{
Treatment with insulin (analogues) and breast cancer risk in diabetics; a systematic review and meta-analysis of in vitro, animal and human evidence
}

Heleen K Bronsveld ${ }^{1,6 \dagger}$, Bas ter Braak ${ }^{2+}$, Øystein Karlstad ${ }^{3}$, Peter Vestergaard ${ }^{4}$, Jakob Starup-Linde ${ }^{4,5}$, Marloes T Bazelier ${ }^{6}$, Marie L De Bruin ${ }^{6}$, Anthonius de Boer ${ }^{6}$, Christine L E Siezen", Bob van de Water ${ }^{2}$, Jan Willem van der Laan²,7,8 and Marjanka K Schmidt ${ }^{*}$

\begin{abstract}
Introduction: Several studies have suggested that anti-diabetic insulin analogue treatment might increase cancer risk. The aim of this study was to review the postulated association between insulin and insulin analogue treatment and breast cancer development, and plausible mechanisms.

Method: A systematic literature search was performed on breast cell-line, animal and human studies using the key words 'insulin analogue' and 'breast neoplasia' in MEDLINE at PubMed, EMBASE, and ISI Web of Science databases. A quantitative and qualitative review was performed on the epidemiological data; due to a limited number of reported estimates, a meta-analysis was performed for glargine only. A comprehensive overview was composed for in vitro and animal studies. Protein and gene expression was analysed for the cell lines most frequently used in the included in vitro studies.
\end{abstract}

Results: In total 16 in vitro, 5 animal, 2 in vivo human and 29 epidemiological papers were included. Insulin AspB10 showed mitogenic properties in vitro and in animal studies. Glargine was the only clinically available insulin analogue for which an increased proliferative potential was found in breast cancer cell lines. However, the pooled analysis of 13 epidemiological studies did not show evidence for an association between insulin glargine treatment and an increased breast cancer risk (HR 1.04; $95 \% \mathrm{Cl} 0.91-1.17 ; \mathrm{p}=0.49$ ) versus no glargine in patients with diabetes mellitus. It has to be taken into account that the number of animal studies was limited, and epidemiological studies were underpowered and suffered from methodological limitations.

Conclusion: There is no compelling evidence that any clinically available insulin analogue (Aspart, Determir, Glargine, Glulisine or Lispro), nor human insulin increases breast cancer risk. Overall, the data suggests that insulin treatment is not involved in breast tumour initiation, but might induce breast tumour progression by up regulating mitogenic signalling pathways.

\footnotetext{
* Correspondence: mk.schmidt@nki.nl

${ }^{\dagger}$ Equal contributors

'Division of Molecular Pathology, The Netherlands Cancer Institute,

Plesmanlaan 121, 1066 CX Amsterdam, The Netherlands

Full list of author information is available at the end of the article
} 


\section{Introduction}

Breast cancer is the most prevalent cancer in women with 1.67 million new cancer cases diagnosed in 2012 worldwide [1]. Diabetes mellitus (DM) has been associated with breast cancer [2]. However, it is unknown if this association is due to the high blood glucose levels of DM, hyperinsulinaemia, shared risks factors such as obesity, or side-effects of diabetic treatment.

Exogenous insulin treatment for diabetics includes animal insulin, human insulin and insulin analogues. Insulin can act as a growth factor, and it is biologically plausible that use of exogenous insulin (analogues), could stimulate neoplastic growth [3]. The initial source of insulin for clinical use in humans was from animal pancreas. Gradually animal insulin has been almost completely replaced by modified or biosynthetic human insulin, such as NPH, Lente or Regular, and insulin analogues. Insulin analogues have been marketed since 1997 and are different from the human insulin molecule in that the amino acid sequence is modified to have an altered pharmacokinetic profile. These modifications afford greater flexibility in the treatment of diabetic patients. However, structural transformation of human insulin might also result in different binding affinity towards the insulin-like growth factor-1 (IGF-1) receptor (IGF1R). This may result in increased mitogenic action of insulin analogues. As each insulin analogue has different alterations in the amino acid sequence, the pharmacologic properties of the analogues are slightly different. Therefore it could be that various insulin analogues have different tumour promoting properties. Glargine is theoretically most likely to have increased mitogenic action compared to human insulin, as the carboxy terminal of the B-chain of glargine has a positive charge, as is the case with IGF-1.

In 2009, the results of four large-scale epidemiological studies were published, raising the concern that insulin analogues, especially insulin glargine, might increase the risk of cancer [4-8]. Two of these studies suggested that insulin glargine may be associated with a higher risk of cancer than treatment with human insulin [5, 8]. Although the results were inconsistent and the authors stressed the limitations of their studies, this led to an urgent call for more research by the European Association for the Study of Diabetes [9].

Previous reviews that focussed on in vitro studies consistently reported that in contrast to other commercially available analogues, glargine has increased binding affinity towards IGF1R. Most studies concluded that glargine may have increased mitogenic potential in particular at supra-physiological concentrations [10, 11]. Extrapolation of these results to humans is difficult due to obvious limitations of in vitro studies, but also due to tissuespecific biological responses. A focus on a specific cancer type could clarify this issue.
The published animal studies on insulin analogues and cancer have not been reviewed so far. In addition, metaanalyses of epidemiological studies have been inconsistent. One meta-analysis reported an increased relative risk (RR) of any cancer among insulin (analogue) users compared to non-insulin-treated diabetics of 1.39 (95\% CI 1.14, 1.70) [12], while another reported no effect (RR 1.04; $95 \%$ CI 0.75, 1.45) [13]. Insulin use was not associated with an increased risk of breast cancer. However, two $[13,14]$ out of four meta-analyses [13-16] concluded that the risk of breast cancer was increased among glargine users compared to non-glargine-users.

Considering that cancer is a heterogeneous disease with different aetiologies, and breast cancer being the most common female cancer, we focussed this review on the association of exogenous insulin (analogue) exposure and the risk of breast cancer. To study breast cancer risk in an in vitro, animal and human setting, we made a distinction between tumour initiation and progression as most in vivo and in vitro studies can only address tumour progression. Furthermore, from the literature review we deducted what is currently known about signalling pathways involved in insulin-induced tumourigenesis. We included all widely prescribed insulin analogues and insulin AspB10 and included in vitro, animal, in vivo human and epidemiological studies. To our knowledge, this is the first review to provide a complete overview (including in vitro, in vivo and epidemiological evidence) on whether and how insulin analogues could affect breast cancer risk in diabetic patients.

\section{Methods}

This systematic review is registered at PROSPERO [17] with the registration number: CRD42012002477 and was developed according to the preferred reporting items for systematic reviews and meta-analyses (PRISMA) guidelines [18], and with guidance from the Cochrane Collaboration handbook [19].

\section{Data sources and searches}

A search of MEDLINE at PubMed, EMBASE, and ISI Web of Science, was performed using key words 'insulin (analogue)' and 'breast cancer' (or similar terms) through July 2014. The full search strategy is described in the electronic supplementary material (Additional file 1: ESM 1).

\section{Study selection}

Eligible studies had to describe effect measures of exogenous insulin (analogue) use on breast cancer development. We included studies with direct (tumour incidence, size, volume, and metastases) or indirect outcomes (cell proliferation, count, and apoptosis, as well as genes and/or proteins explaining mechanisms of breast cancer tumour 
development e.g., mitogen-activated protein kinase (MAPK), phosphatidylinositol-4,5-bisphosphate 3-kinase (PI3K), phosphatase and tensin homolog (PTEN), mechanistic target of rapamycin mTOR (p53), that are associated with breast cancer. Studies were divided into three categories with the following selection criteria; 1) in vitro studies of mammary gland cell lines exposed to insulin analogues, in which direct proliferative effect was measured or pathway activation was monitored; 2) animal studies on models treated with insulin analogue, in which the mammary gland tumour progression/initiation was measured, or different insulin analogues were compared for their activation of mitogenic signalling pathways in mammary gland tissue, and 3) epidemiological and in vivo studies in humans, including patients with type 1 or type $2 \mathrm{DM}$ treated with insulin analogues before breast cancer diagnosis; cohort and case-control studies as well as randomized controlled trials were included. Only epidemiological studies that presented relative or absolute risk estimates for breast cancer among insulin users were included. Studies that used a non-DM reference population were excluded. In case of multiple publications on the same dataset, we included the study with most complete data. An overview of the study selection is provided in Fig. 1.

\section{Data extraction}

For the in vitro and animal studies information was extracted on the cell (with insulin receptor (INSR):IGF1R status) or animal model (species, tumour subtype), study design (in vitro: assay, starvation method, exposure time, type and refreshment of medium, and presence of

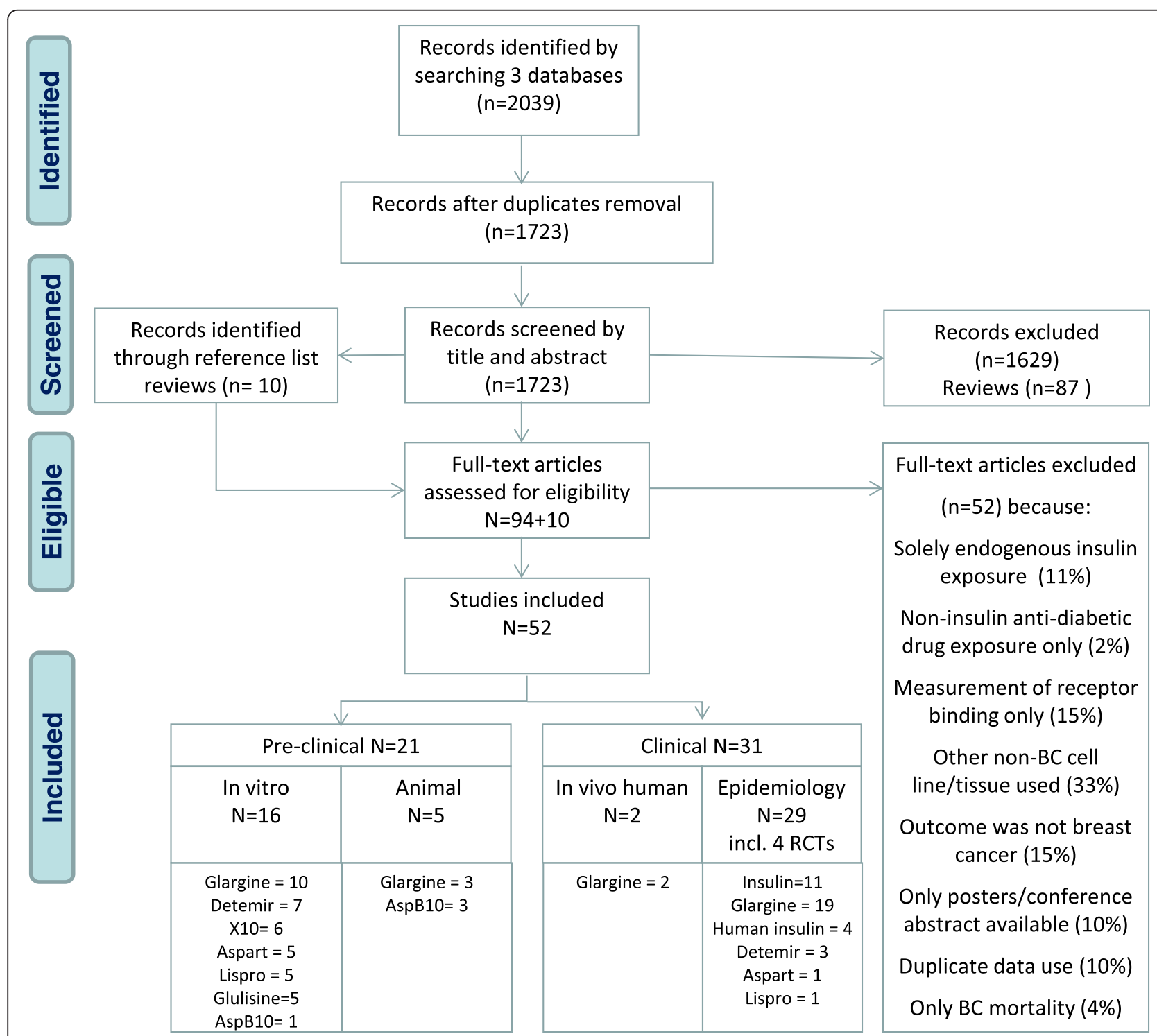

Fig. 1 Flow chart of study identification and study selection process. $B C$ breast cancer 
phenol red; animal: tissue and proteins analysed, and time of sampling), the intervention (compounds and concentration/dose tested) and the study outcome (mammary tumour formation, mitogenic response, and pathway activation) (Tables 1 and 2).

For each epidemiological study, information was extracted on study design and characteristics, i.e., country, source population, data sources, study period, age group, matching variables for case-control studies, DM type and definition, prevalent/incident insulin users, exposure definition, time of exposure definition, mean duration of exposure, latency period and covariates (Additional file 1: Table S2, S3c), and risk estimates for each exposure comparison (Table 3).

\section{Data synthesis and analyses}

In vitro and animal studies were grouped by type of insulin analogue, and common pathways/mechanisms of action were extracted and summarized. Plausible pathways were suggested based on the strength of the evidence. To substantiate the results of the in vitro studies included in this systematic review, we created an overview of the protein and gene expression in eight commonly used mammary (tumour) cell lines of hormone receptor levels (INSR, IGF1R, ER, PR, HER2, EGFR) and some proteins essential for insulin-induced downstream signalling cascades. The methods of these experiments can be found in Additional file 1: ESM 2.

The exposure comparisons that were examined in the epidemiological studies were categorized as: 1) use of any exogenous insulin versus no use of any exogenous insulin (drug exposure undefined); 2) use of any exogenous insulin versus use of non-insulin anti-diabetic drug (NIAD) (type of NIAD defined); 3) use of insulin X versus no use of insulin X. Results were categorized on the exposure of interest. Data was ordered per risk estimate (hazard ratio (HR), odds ratio (OR), incidence rate ratio (IRR)). If a study presented results within the same exposure comparison, but with different definitions of the exposure of interest (e.g., glargine users or glargine only users), the group that had most power was included to calculate the pooled estimate. We set a subjective cutoff of 10 studies needed for a pooled analysis; hence, this was only performed for glargine. The pooled estimate was derived using the random effect model. Pooled analysis by dose or duration was not feasible, as risk estimates were reported for different exposure comparisons, exposure definitions (e.g., mean or cumulative dose, duration since start exposure, or cumulative duration) and stratification categories. The quality evaluation of the epidemiological studies focussed on potential selection bias, information bias, and confounding. The evaluation process of the bias and power of studies is displayed in
Additional file 1: ESM 3. Data were prepared in Microsoft Access 2010 and analysed in Stata version 11.0.

\section{Results}

A search in MEDLINE at PubMed, EMBASE, and ISI Web of Science identified 1,723 unique records (Fig. 1). After the eligibility assessment, 52 studies on exogenous insulin (analogue) exposure and breast cancer were included, of which there were 16 in vitro, 5 animal, 2 human in vivo and 29 epidemiological studies (see Additional file 1: ESM 4 for study descriptions).

\section{Evidence of mitogenic/carcinogenic potential}

Current evidence of the mitogenic/carcinogenic potential per insulin (analogue) is described below, highlighting the most important findings displayed in the tables and figures. In Table 1 an overview is presented of all in vitro studies in which the mitogenic potency and/or stimulation of the signalling pathways MAPK and PI3K upon insulin analogue(s) exposure was determined in a mammary gland (tumour) cell line [20-35]. Protein expression of hormone receptors and some downstream signalling proteins for each cell line are provided in Additional file 1: Table S1 and Fig. 2. In Table 2 an overview is presented of all relevant animal studies [36-40]. Descriptions and characteristics of the epidemiological studies are presented in Additional file 1: Table S2, S3c [5, 6, 41-67]. Table 3 lists the overall risk estimates for breast cancer per insulin analogue in the epidemiological studies; the corresponding forest plots are presented in Additional file 1: Figure S1. Results of the meta-analysis on glargine can be found in Fig. 3. Some studies provided risk estimates by strata of duration or dose of exposure (Additional file 1: Table S4). The quality assessment of the epidemiological studies is shown in Additional file 1: Table S5.

\section{Insulin glargine (M1/M2)}

Seven of ten in vitro studies found an increased proliferative potential of glargine in comparison with human insulin [22, 25, 28, 29, 31, 34, 35] (Table 1). Two studies found proliferation of glargine as well, but human insulin was not included as a reference compound, therefore they could not confirm an increased proliferative response [32, 33]. One study is difficult to interpret, because IGF1 did not show increased mitogenic potential either [24]. Similar to insulin AspB10, glargine has an increased binding affinity towards IGF1R [68]. This receptor is assumed to be responsible for the increased mitogenic action. Studies including kinase activation assays indicate that the PI3K signalling cascade is significantly upregulated after glargine stimulation compared to human insulin stimulation $[28,31,33,34]$. Two studies also found the MAPK signalling cascade to be 
Table 1 Overview of in vitro studies in breast cancer cell lines on the mitogenic potential of insulin analogues

\begin{tabular}{|c|c|c|c|c|c|c|c|c|c|c|c|c|c|c|}
\hline Author, year & Cell line & $\begin{array}{l}\text { INSR/ } \\
\text { IGF1R }\end{array}$ & Method & Starvation & $\begin{array}{l}\text { Stimulation } \\
\text { time }\end{array}$ & $\begin{array}{l}\text { Refreshment } \\
\text { of medium }\end{array}$ & $\begin{array}{l}\text { Type of } \\
\text { stimulation } \\
\text { medium }\end{array}$ & $\begin{array}{l}\text { Presence } \\
\text { phenol } \\
\text { red }\end{array}$ & $\begin{array}{l}\text { Analogues } \\
\text { tested }\end{array}$ & $\begin{array}{l}\text { Concentrations } \\
\text { tested } \mathrm{nM}\end{array}$ & $\begin{array}{l}\text { Mitogenic } \\
\text { response }\end{array}$ & Sig. & $\begin{array}{l}\text { PI3K } \\
\text { pathway* }\end{array}$ & $\begin{array}{l}\text { MAPK } \\
\text { pathway* }\end{array}$ \\
\hline \multirow[t]{3}{*}{$\begin{array}{l}\text { Milazzo et al., } \\
1997 \text { [26] }\end{array}$} & \multirow[t]{2}{*}{ MCF7 $^{A}$} & $1: 4$ & $\begin{array}{l}{[3 \mathrm{H}] \text { Thymidine }} \\
\text { incorporation }\end{array}$ & Yes & $\begin{array}{l}24 \text { hrs stim } \\
2 \text { hrs } \\
\text { measure }\end{array}$ & Yes & $\begin{array}{l}\text { MEM DME/ } \\
\text { F12 + } 0.1 \% \\
\text { BSA }\end{array}$ & Yes & AspB10 & 10 & $\uparrow^{A, B}$ & Yes & & \\
\hline & & \multirow[t]{2}{*}{$1: 0.8$} & DNA measurement & Yes & $3-5$ days & $\begin{array}{l}\text { Yes, every } \\
\text { two days }\end{array}$ & $\begin{array}{l}\text { MEM DME/ } \\
\text { F12 + } 0.1 \% \\
\text { BSA }\end{array}$ & Yes & AspB10 & $0.01-10$ & $\uparrow^{A, B}$ & yes & & \\
\hline & MCF $10^{B}$ & & Colony forming assay & No & 2 weeks & $\begin{array}{l}\text { Yes, every } \\
\text { two days }\end{array}$ & $\begin{array}{l}\text { MEM DME/ } \\
\text { F12 + } 2 \% \\
\text { BSA }\end{array}$ & Yes & AspB10 & 100 & $\uparrow^{A}-^{B}$ & Yes & & \\
\hline \multirow[t]{3}{*}{$\begin{array}{l}\text { Staiger et al., } \\
2007 \text { [32] }\end{array}$} & MCF7 $^{A}$ & - & $\begin{array}{l}{[3 \mathrm{H}] \text { Thymidine }} \\
\text { incorporation }\end{array}$ & $48 h^{\mathrm{A}}$ & $\begin{array}{l}20 \text { hrs stim } \\
4 \text { hrs } \\
\text { measure }\end{array}$ & Yes & $\begin{array}{l}\text { DME/F12 } \\
\text { SFM }\end{array}$ & No & Glargine & $10,50,100$ & $\downarrow^{A}$ & No & & \\
\hline & \multirow[t]{2}{*}{ MCF $10^{B}$} & \multirow[t]{2}{*}{-} & \multirow[t]{2}{*}{ MTT } & $24 h^{B}$ & 4 days & \multirow{2}{*}{$\begin{array}{l}\text { Yes, every } \\
\text { two days }\end{array}$} & \multirow{2}{*}{$\begin{array}{l}\text { DME/F12 } \\
\text { SFM }\end{array}$} & \multirow[t]{2}{*}{ No } & \multirow[t]{2}{*}{ Glargine } & \multirow[t]{2}{*}{$1,5,10,25$} & \multirow[t]{2}{*}{$\uparrow^{A, B}$} & \multirow[t]{2}{*}{ No } & & \\
\hline & & & & No & & & & & & & & & & \\
\hline \multirow{2}{*}{$\begin{array}{l}\text { Liefvendahl } \\
\text { et al., } 2008 \\
\text { [24] }\end{array}$} & \multirow{2}{*}{$\begin{array}{l}\text { MCF7 } \\
\text { SKBR-3 }\end{array}$} & $1: 20$ & \multirow{2}{*}{$\begin{array}{l}{[3 \mathrm{H}] \text { Thymidine }} \\
\text { incorporation }\end{array}$} & \multirow[t]{2}{*}{$24 \mathrm{hrs}$} & \multirow{2}{*}{$\begin{array}{l}21 \text { hrs stim } \\
3 \text { hrs } \\
\text { measure }\end{array}$} & \multirow[t]{2}{*}{ No } & \multirow[t]{2}{*}{ DMEM SFM } & \multirow[t]{2}{*}{ No } & \multirow[t]{2}{*}{ Glargine } & \multirow[t]{2}{*}{$0.01-100$} & \multirow[t]{2}{*}{-} & & & \\
\hline & & $1: 1.8$ & & & & & & & & & & & & \\
\hline \multirow{5}{*}{$\begin{array}{l}\text { Mayer et al., } \\
2008 \text { [25] }\end{array}$} & \multirow[t]{2}{*}{ MCF7 $^{A}$} & \multirow[t]{2}{*}{ 1:3 } & \multirow[t]{5}{*}{ Cristal violet cell staining } & No & 4 days & No & DMEM + 1 & No & Aspart & $1.5^{\mathrm{A}, \mathrm{B}}$ & & & & \\
\hline & & & & & & & $\%$ SD-FBS & & Lispro & $15^{A, B}$ & & & & \\
\hline & MCF10A ${ }^{B}$ & $1: 1.2$ & & & & & & & Glargine & $1500^{c}$ & $\uparrow^{A}$ & Yes $^{\mathrm{A}}$ & & \\
\hline & & & & & & & & & Glulisine & & & & & \\
\hline & $\mathrm{T} 47 \mathrm{D}^{\mathrm{C}}$ & $1: 2$ & & & & & & & Detemir & & & & & \\
\hline Shukla et al., & MCF7 $^{\mathrm{A}}$ & - & Cristal violet cell staining & $24 \mathrm{hrs}$ & 3 days $^{A}$ & Yes, every 24 & DMEM + 2 & No & Aspart & $1.5,15,150$ & $\uparrow^{A}$ & No & & \\
\hline & & & & & & & \% CDFBS & & Lispro & 1500 & - & & & \\
\hline & & & & & 2 days $^{B}$ & & $\mathrm{MEGM}^{B}$ & & Glargine & & $\uparrow^{A}$ & yes & & \\
\hline & & & & & & & & & Detemir & & $\downarrow^{\mathrm{A}}$ & No & & \\
\hline & MCF10A ${ }^{B}$ & - & WB & $24 \mathrm{hrs}$ & $10 \mathrm{~min}$ & - & DMEM + 2 & No & Aspart & & & & - & - \\
\hline & & & & & & & $\%$ CDFBS & & Lispro & & & & - & - \\
\hline & & & & & & & MEGM $^{B}$ & & Glargine & & & Yes & $\uparrow^{A, B}$ & $\uparrow^{A}$ \\
\hline & & & & & & & & & Detemir & & & Yes & $\downarrow^{\mathrm{A}}$ & - \\
\hline $\begin{array}{l}\text { Shukla et al., } \\
2009 \text { [30] }\end{array}$ & MCF7 $^{A}$ & - & Cristal violet cell staining & $24 \mathrm{hrs}$ & 3 days $^{A}$ & $\begin{array}{l}\text { Yes, every } 24 \\
\text { hrs }\end{array}$ & $\begin{array}{l}\text { DMEM + } 2 \\
\% \text { CDFBS }\end{array}$ & No & Glulisine & $\begin{array}{l}1.5,15,150 \\
1500\end{array}$ & $\downarrow^{A B}$ & No & & \\
\hline & MCF10 ${ }^{B}$ & - & & & 2 days $^{B}$ & & MEGM & & & & & & & \\
\hline & & & $\begin{array}{l}\text { MMOC/ki67 nuclei } \\
\text { count }\end{array}$ & No & 3 days & No & $\begin{array}{l}\text { Waymouth } \\
\text { medium } \\
\text { SFM }\end{array}$ & & Glulisine & 750 & $\downarrow$ & No & & \\
\hline
\end{tabular}


Table 1 Overview of in vitro studies in breast cancer cell lines on the mitogenic potential of insulin analogues (Continued)

\begin{tabular}{|c|c|c|c|c|c|c|c|c|c|c|c|c|c|c|}
\hline & & & WB & $24 \mathrm{hrs}$ & $10 \mathrm{~min}$ & - & $\begin{array}{l}\text { DMEM + } 2 \\
\% \text { CDFBS }^{A}\end{array}$ & No & Glulisine & & & Yes & $\downarrow^{A B}$ & $\downarrow^{A B}$ \\
\hline & & & & & & & MEGM ${ }^{B}$ & & & & & & & \\
\hline \multirow{2}{*}{$\begin{array}{l}\text { Weinstein } \\
\text { et al., } 2010 \\
\text { [35] }\end{array}$} & \multirow[t]{2}{*}{ MCF7 } & \multirow[t]{2}{*}{-} & \multirow[t]{2}{*}{ Cell counting } & \multirow[t]{2}{*}{ No } & \multirow[t]{2}{*}{72 hrs } & \multirow{2}{*}{$\begin{array}{l}\text { Yes every } \\
\text { day }\end{array}$} & \multirow[t]{2}{*}{ DMEM/SFM } & & Glargine & 100 & $\uparrow$ & No & & \\
\hline & & & & & & & & & Detemir & & $\uparrow$ & No & & \\
\hline \multirow{2}{*}{$\begin{array}{l}\text { Oleksiewicz } \\
\text { et al., } 2011 \\
\text { [27] }\end{array}$} & \multirow[t]{2}{*}{ MCF7 } & \multirow[t]{2}{*}{-} & FACS & 72 hrs & 24-30 hrs & No & $\begin{array}{l}\text { DMEM + } 0.1 \\
\% \text { FCS }\end{array}$ & No & $\mathrm{X} 10$ & $0.074-2$ & $\uparrow$ & Yes & & \\
\hline & & & WB & 72 hrs & $20-40 \mathrm{~min}$ & No & $\begin{array}{l}\text { DMEM + } 0.1 \\
\% \text { FCS }\end{array}$ & No & $\times 10$ & $0.67,2$ & & Yes & $\uparrow$ & $\uparrow$ \\
\hline \multirow{4}{*}{$\begin{array}{l}\text { Teng et al., } \\
2011 \text { [33] }\end{array}$} & \multirow[t]{4}{*}{$\mathrm{MCF}^{\mathrm{A}}$} & \multirow[t]{4}{*}{-} & \multirow[t]{2}{*}{ MTT } & \multirow[t]{2}{*}{24 hours } & \multirow[t]{2}{*}{2 days } & \multirow{2}{*}{$\begin{array}{l}\text { Yes, every } \\
\text { two days }\end{array}$} & \multirow{2}{*}{$\begin{array}{l}\text { RPMI + } 0.5 \\
\% \text { CS-FBS }\end{array}$} & \multirow[t]{2}{*}{ No } & \multirow[t]{2}{*}{ Glargine } & \multirow[t]{2}{*}{$20-200$} & \multirow[t]{2}{*}{$\uparrow^{A}$} & Yes & & \\
\hline & & & & & & & & & & & & Yes & & \\
\hline & & & WB & No & $\begin{array}{l}0,30,60 \\
120,240 \\
\min \end{array}$ & No & $\begin{array}{l}\text { RPMI + } 0.5 \\
\% \text { CS-FBS }\end{array}$ & No & Glargine & $100 \mathrm{nM}$ & $\uparrow^{A}$ & & & \\
\hline & & & FACS anti-apoptotic & No & $48 \mathrm{hrs}$ & No & $\begin{array}{l}\text { RPMI + } 0.5 \\
\% \text { CS-FBS }\end{array}$ & & Glargine & & $\begin{array}{l}\uparrow^{A} \text { anti- } \\
\text { Apoptotic } \\
\text { response }\end{array}$ & Yes & & \\
\hline \multirow{10}{*}{$\begin{array}{l}\text { Glendorf } \\
\text { et al., } 2012 \\
\text { [21] }\end{array}$} & \multirow[t]{10}{*}{ HMEC } & \multirow[t]{10}{*}{$1: 20$} & \multirow{10}{*}{$\begin{array}{l}{[3 \mathrm{H}] \text { Thymidine }} \\
\text { incorporation }\end{array}$} & \multirow[t]{10}{*}{ No } & 70 hrs stim & No & MEGM & $?$ & B10A, & $0.0001-1000$ & $\downarrow$ & & & \\
\hline & & & & & $\begin{array}{l}2 \text { hrs } \\
\text { measure }\end{array}$ & & & & $\mathrm{B} 10 \mathrm{R}$ & & $\downarrow$ & & & \\
\hline & & & & & & & & & $\mathrm{X} 10$ & & $\uparrow$ & & & \\
\hline & & & & & & & & & $\mathrm{B} 10 \mathrm{Q}$, & & $\uparrow$ & & & \\
\hline & & & & & & & & & $\mathrm{B} 10 \mathrm{E}$ & & $\uparrow$ & & & \\
\hline & & & & & & & & & $\mathrm{B} 10 \mathrm{H}$ & & $\downarrow$ & & & \\
\hline & & & & & & & & & B10l, & & $\downarrow$ & & & \\
\hline & & & & & & & & & $\mathrm{B} 10 \mathrm{~F}$ & & $\downarrow$ & & & \\
\hline & & & & & & & & & B10W, & & $\downarrow$ & & & \\
\hline & & & & & & & & & B10V & & $\downarrow$ & & & \\
\hline Hansen et al., & $\mathrm{HMEC}^{\mathrm{A}}$ & $1: 21$ & {$[3 \mathrm{H}]$ Thymidine } & $24 \mathrm{hrs}$ & 70 hrs stim & No & MEGM & No & Detemir & $0.001-1000$ & $\downarrow^{\mathrm{A}}$ & Yes & & \\
\hline & & & & & $\begin{array}{l}2 \text { hrs } \\
\text { measure }\end{array}$ & & & & Glargine & & $\uparrow^{A}$ & Yes & & \\
\hline & & & & & & & & & $\times 10$ & & $\uparrow^{A}$ & Yes & & \\
\hline $\begin{array}{l}\text { Knudsen } \\
\text { et al., } 2012 \\
\text { [23] }\end{array}$ & $\mathrm{MCF}^{\mathrm{A}}$ & - & $\begin{array}{l}{[3 \mathrm{H}] \text { Thymidine }} \\
\text { incorporation }\end{array}$ & 2 hrs & $\begin{array}{l}24 \text { hrs stim } \\
2 \text { hrs } \\
\text { measure }\end{array}$ & No & $\begin{array}{l}\text { DMEM + } 0.1 \\
\% \text { serum }\end{array}$ & No & S961 & $0.0001-100$ & $\uparrow^{A}$ & & & \\
\hline $\begin{array}{l}\text { Pierre- } \\
\text { Eugene et al., }\end{array}$ & $\mathrm{MCF}^{\mathrm{A}}$ & - & BRET-PIP $_{3}$ & No & $45 \mathrm{~min}$ & No & $\begin{array}{l}\text { DMEM/F12 } \\
+5 \% \text { FBS }\end{array}$ & $?$ & Aspart & & & & - & \\
\hline $2012[28]$ & MDA- & - & & & & & & & Lispro & & & & - & \\
\hline & $M B-231^{\circ}$ & & & & & & & & Glargine & & & Yes & $\uparrow^{A}$ & \\
\hline
\end{tabular}


Table 1 Overview of in vitro studies in breast cancer cell lines on the mitogenic potential of insulin analogues (Continued)

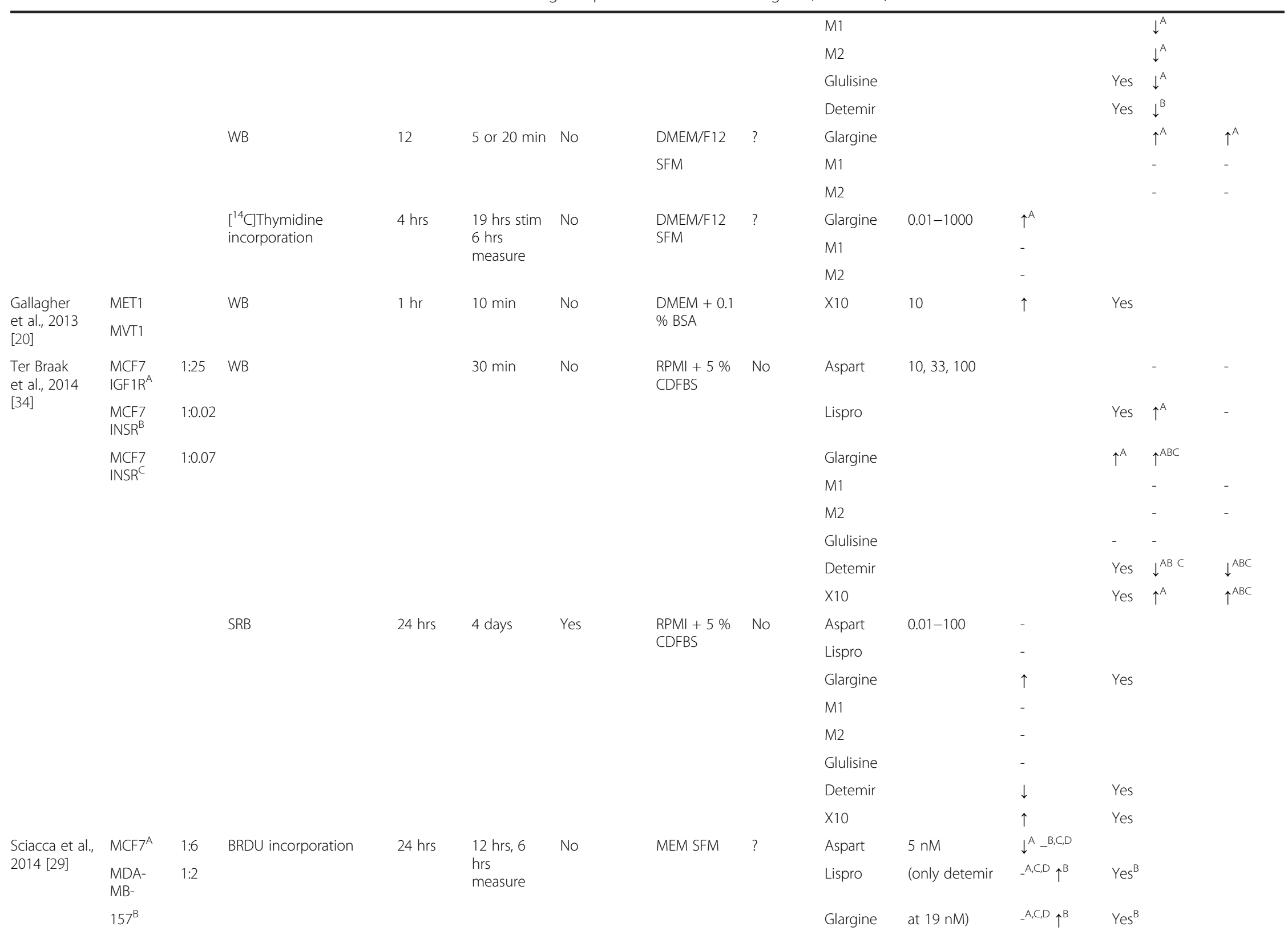


Table 1 Overview of in vitro studies in breast cancer cell lines on the mitogenic potential of insulin analogues (Continued)

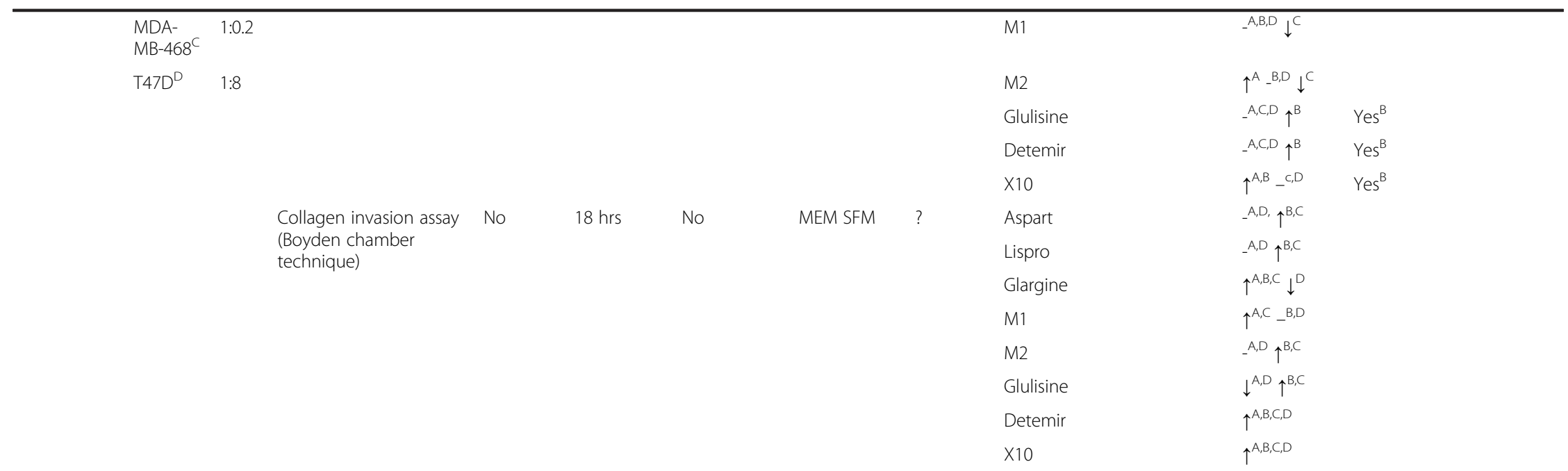

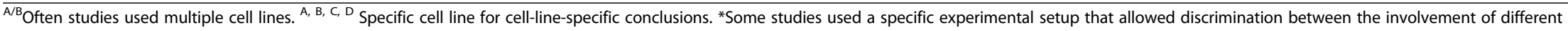
pathways. For all these studies the p-ERK and p-AKT served as biomarker for activation of mitogen-activated protein kinase (MAPK) or phosphatidylinositol-4,5-bisphosphate 3-kinase (PI3K), respectively. IGF1R insulinlike growth factor-1 receptor, BRDU 5-Bromo-2'-deoxyuridine, RPMI Roswell Park Memorial Institute medium, MTT Microculture Tetrazolium proliferation Assay, WB Western Blot, BRET-PIP Bioluminescence Resonance Energy Transfer assay in which the phophatidylinositol-3 phosphate (PIP(3)) production was monitored, SRB SulfoRhodamine B proliferation assay, MEGM Mammary Epithelial Cell Growth Medium, MEM Minimum Essential Medium, SFM Serum Free Medium, CDFBS Charcoal-Dextran-Treated Fetal Bovine Serum, Sig Significant. 
Table 2 Overview of in vivo studies in animals on the correlation of insulin analogues and breast cancer

\begin{tabular}{|c|c|c|c|c|c|c|c|c|c|c|c|}
\hline Author, year & Model & $\begin{array}{l}\text { Number of } \\
\text { animals per } \\
\text { treatment } \\
\text { group }\end{array}$ & $\begin{array}{l}\text { Tissues } \\
\text { analysed }\end{array}$ & $\begin{array}{l}\text { Time points } \\
\text { sampling }\end{array}$ & $\begin{array}{l}\text { Analogues } \\
\text { tested }\end{array}$ & $\begin{array}{l}\text { Dose } \\
\text { tested } \\
\text { nM }\end{array}$ & Method & $\begin{array}{l}\text { Proteins } \\
\text { analysed }\end{array}$ & $\begin{array}{l}\text { Carcinogenic } \\
\text { potential }\end{array}$ & Sig. & Tumour characteristics \\
\hline $\begin{array}{l}\text { Stammberger et al., } \\
2002 \text { [37] (re- } \\
\text { evaluation in 2012) } \\
\text { [38] }\end{array}$ & $\begin{array}{l}\text { Sprague-Dawley rats } \\
\text { and Wistar rats and } \\
\text { NMRI mice }\end{array}$ & $5-30$ & $\begin{array}{l}\text { No further } \\
\text { tumour } \\
\text { characterisation }\end{array}$ & $\begin{array}{l}\text { Follow up of } 2 \\
\text { years }\end{array}$ & Glargine & $\begin{array}{l}2,5 \\
12.5 \\
\mathrm{IU} / \mathrm{Kg}\end{array}$ & $\begin{array}{l}\text { Spontaneous } \\
\text { mammary gland } \\
\text { tumour formation } \\
\text { upon treatment }\end{array}$ & & - & & \multirow[t]{9}{*}{$\begin{array}{l}\text { MG adenoma, } \\
\text { fibroadenoma, } \\
\text { adenocarcinoma }\end{array}$} \\
\hline \multirow[t]{6}{*}{$\begin{array}{l}\text { Gallagher et al., } \\
2012 \text { [36] }\end{array}$} & \multirow{6}{*}{$\begin{array}{l}\text { Orthotopic mammary } \\
\text { tumour weight and } \\
\text { hyperinsulinaemic MKR } \\
\text { mice }\end{array}$} & \multirow[t]{6}{*}{$3-4$} & $\begin{array}{l}\text { Mammary } \\
\text { gland }\end{array}$ & \multirow[t]{6}{*}{$0-25$ days } & \multirow[t]{6}{*}{ AspB10 } & $\begin{array}{l}12.5 \\
\mathrm{IU} / \mathrm{kg}\end{array}$ & $\begin{array}{l}\text { Tumour volume } \\
\text { measurement }\end{array}$ & & $\uparrow$ & Yes & \\
\hline & & & $\begin{array}{l}\text { Lung } \\
\text { metastasis }\end{array}$ & & & $2 x /$ day & $\begin{array}{l}\text { Counting lung } \\
\text { metastases }\end{array}$ & & $\uparrow$ & No & \\
\hline & & & & & & & \multirow[t]{4}{*}{ WB receptor activation } & $p-I N S R$ & $\uparrow$ & Yes & \\
\hline & & & & & & & & p-IGF1R & & & \\
\hline & & & & & & & & p-Akt & $\uparrow$ & Yes & \\
\hline & & & & & & & & $p$-Erk & - & & \\
\hline \multirow[t]{2}{*}{$\begin{array}{l}\text { Tennagels et al., } \\
2013 \text { [39] }\end{array}$} & \multirow[t]{2}{*}{$\begin{array}{l}\text { Female Sprague-Dawley } \\
\text { rats }\end{array}$} & \multirow[t]{2}{*}{$3-4$} & \multirow[t]{2}{*}{$\begin{array}{l}\text { Mammary } \\
\text { gland }\end{array}$} & \multirow[t]{2}{*}{$60 \mathrm{~min}$} & Glargine & $\begin{array}{l}12.5 \\
\mathrm{U} / \mathrm{kg}\end{array}$ & \multirow[t]{2}{*}{ WB kinase activation } & $p-I N S R$ & - & & \\
\hline & & & & & AspB10 & & & p-IGF1R & $\uparrow$ & Yes & \\
\hline \multirow[t]{11}{*}{$\begin{array}{l}\text { Ter Braak et al., } \\
2015 \text { [40] }\end{array}$} & \multirow[t]{11}{*}{$\begin{array}{l}\mathrm{p} 53^{\mathrm{R} 270 \mathrm{H} /+} \text { WAPCre FVB } \\
\text { mice }\end{array}$} & \multirow[t]{11}{*}{40} & \multirow[t]{11}{*}{$\begin{array}{l}\text { Mammary } \\
\text { gland tumors }\end{array}$} & \multirow[t]{11}{*}{$\begin{array}{l}\text { Chronic } \\
\text { exposure till } \\
\text { MG tumor } \\
\text { development }\end{array}$} & Glargine & $\begin{array}{l}12.5- \\
15 \mathrm{IU} / \\
\mathrm{kg}\end{array}$ & \multirow[t]{2}{*}{ Tumour latency time } & & $\uparrow$ & No & \multirow{11}{*}{$\begin{array}{l}\text { Majority aggressive } \\
\text { EMT no correlation } \\
\text { pathology and } \\
\text { treatment }\end{array}$} \\
\hline & & & & & AspB10 & $\begin{array}{l}150- \\
200 \\
\mathrm{IU} / \mathrm{kg}\end{array}$ & & INSR & $\uparrow$ & Yes & \\
\hline & & & & & & & \multirow{9}{*}{$\begin{array}{l}\text { WB protein expression } \\
\text { profiling }\end{array}$} & IGF1R, & & & \\
\hline & & & & & & & & Erk, & & & \\
\hline & & & & & & & & p-Erk & $\uparrow$ & Yes & \\
\hline & & & & & & & & Akt, & & & \\
\hline & & & & & & & & p-Akt, & $\uparrow$ & Yes & \\
\hline & & & & & & & & EGFR, & & & \\
\hline & & & & & & & & $E R$ & & & \\
\hline & & & & & & & & E-cad, & & & \\
\hline & & & & & & & & $\begin{array}{l}\text { N-cad, } \\
\text { Her2 }\end{array}$ & & & \\
\hline
\end{tabular}


Table 3 Relative risk estimations for breast cancer among insulin treatment groups and the evaluation of bias and power of the studies

\begin{tabular}{|c|c|c|c|c|c|c|c|c|}
\hline Author, year & Exposure of interest & $\begin{array}{l}\text { Exposure comparison } \\
\text { group }\end{array}$ & $\begin{array}{l}\text { Cases/controls*** or cases/person- } \\
\text { years }\end{array}$ & $\begin{array}{l}\text { Cases/controls }{ }^{* * *} \text { or cases/person- } \\
\text { years****in comparison group (number) }\end{array}$ & $\begin{array}{l}\text { Risk } \\
\text { Ratio** }\end{array}$ & $\begin{array}{l}95 \% \\
\mathrm{Cl}\end{array}$ & $\begin{array}{l}\text { Risk of } \\
\text { bias }\end{array}$ & Power \\
\hline \multicolumn{9}{|l|}{$\begin{array}{l}\text { Any insulin-no insulin: } \\
\text { hazard ratio }\end{array}$} \\
\hline $\begin{array}{l}\text { Carstensen et al., } \\
2012 \text { [43] }\end{array}$ & Insulin users & No insulin users & $248 / 102,500$ & $2,118 / 627,100$ & 0.96 & $\begin{array}{l}0.84 \\
1.09\end{array}$ & Moderate & Adequate \\
\hline $\begin{array}{l}\text { Ferrara et al., } 2011 \\
\text { [48] }\end{array}$ & Insulin users & No insulin users & NR & NR & 1.0 & $\begin{array}{l}0.9, \\
1.2\end{array}$ & Moderate & Adequate \\
\hline $\begin{array}{l}\text { Neumann et al., } 2012 \\
\text { [60] }\end{array}$ & Insulin users & No insulin users & $\mathrm{NR} / \mathrm{NR}^{*}$ & $\mathrm{NR} / \mathrm{NR}^{*}$ & 0.86 & $\begin{array}{l}0.81 \\
0.91\end{array}$ & High & Adequate \\
\hline $\begin{array}{l}\text { Onitilo et al., } 2014 \\
\text { [61] }\end{array}$ & Insulin users & No insulin users & $N R / N R^{*}$ & $\mathrm{NR} / \mathrm{NR}^{*}$ & 0.84 & $\begin{array}{l}0.58 \\
1.23\end{array}$ & High & Too low \\
\hline \multicolumn{9}{|l|}{$\begin{array}{l}\text { Any insulin-no insulin: } \\
\text { odds ratio }\end{array}$} \\
\hline $\begin{array}{l}\text { Bodmer et al., 2010a } \\
\text { [41] }\end{array}$ & Insulin users & No insulin users & $43 / 131$ & $262 / 1,022$ & $\mathrm{NE}$ & $N E$ & High & Too low \\
\hline $\begin{array}{l}\text { Cleveland et al., } 2012 \\
\text { [45] }\end{array}$ & Insulin users & No insulin users & $20 / 16$ & $50 / 49$ & 1.15 & $\begin{array}{l}0.40 \\
3.40\end{array}$ & High & Too low \\
\hline \multicolumn{9}{|l|}{$\begin{array}{l}\text { Any insulin-NIAD: } \\
\text { hazard ratio }\end{array}$} \\
\hline Currie et al., 2009a [6] & Insulin users & Metformin only & $N R / 12,640^{*}$ & $\mathrm{NR} / 34,847^{*}$ & 1.07 & $\begin{array}{l}0.79 \\
1.44\end{array}$ & Moderate & Too low \\
\hline $\begin{array}{l}\text { Redaniel et al., 2012a } \\
\text { [62] }\end{array}$ & $\begin{array}{l}\text { Insulin and NIAD } \\
\text { users }\end{array}$ & Sulfonylurea only users & $33 / 8,233.8$ & $93 / 27,308.2$ & 1.23 & $\begin{array}{l}0.63 \\
2.38\end{array}$ & Low & Too low \\
\hline $\begin{array}{l}\text { Redaniel et al., 2012b } \\
\text { [62] }\end{array}$ & Insulin only users & Sulfonylurea only users & $8 / 2,247.3$ & $93 / 27,308.2$ & 1.67 & $\begin{array}{l}0.70 \\
3.99\end{array}$ & Low & Too low \\
\hline $\begin{array}{l}\text { Vallarino et al., } \\
2013^{* * * * * *}[67]\end{array}$ & $\begin{array}{l}\text { Pioglitzone users, } \\
\text { not using insulin }\end{array}$ & $\begin{array}{l}\text { Insulin users, not using } \\
\text { pioglitazone }\end{array}$ & $181 / 29,721$ & $113 / 13,680$ & 0.85 & $\begin{array}{l}0.67 \\
1.08\end{array}$ & High & Low \\
\hline \multicolumn{9}{|l|}{$\begin{array}{l}\text { Any insulin-NIAD: } \\
\text { odds ratio }\end{array}$} \\
\hline Hsieh et al., 2012 [53] & Insulin only users & Metformin only users & $5 / N R$ & 19/NR & 1.63 & $\begin{array}{l}0.60 \\
4.40\end{array}$ & High & Too low \\
\hline Koro et al., 2007a [54] & $\begin{array}{l}\text { Insulin and NIAD } \\
\text { users }\end{array}$ & TZD users & $13 / 52$ & $83 / 449$ & 0.71 & $\begin{array}{l}0.36 \\
1.37\end{array}$ & High & Too low \\
\hline Koro et al., 2007b [54] & Insulin only users & TZD users & $9 / 62$ & $83 / 449$ & 1.27 & $\begin{array}{l}0.61 \\
2.67\end{array}$ & High & Too low \\
\hline \multicolumn{9}{|l|}{$\begin{array}{l}\text { Glargine-no glargine: } \\
\text { hazard ratio }\end{array}$} \\
\hline \multirow[t]{2}{*}{$\begin{array}{l}\text { Bordeleau et al., } \\
2014^{* * * * *}[42]\end{array}$} & Glargine users & $\begin{array}{l}\text { Standard care, not } \\
\text { using glargine }\end{array}$ & $28 / 11,620^{*}$ & $28 / 12,845^{*}$ & 1.15 & $\begin{array}{l}0.67 \\
1.97\end{array}$ & Low & Too low \\
\hline & Glargine users & & $4 / 4,711$ & $6 / 4,524$ & 0.62 & & Moderate & Too low \\
\hline
\end{tabular}


Table 3 Relative risk estimations for breast cancer among insulin treatment groups and the evaluation of bias and power of the studies (Continued)

\begin{tabular}{|c|c|c|c|c|c|c|c|c|}
\hline $\begin{array}{l}\text { Home and } \\
\text { Lagarenne, 2009***** } \\
{[52]}\end{array}$ & & $\begin{array}{l}\text { Any anti-diabetic drug, } \\
\text { NPH in } 20 \text { studies }\end{array}$ & & & & $\begin{array}{l}0.17 \\
2.18\end{array}$ & & \\
\hline $\begin{array}{l}\text { Rosenstock et al., } \\
2009 \text { [63] }\end{array}$ & Glargine users & $\mathrm{NPH}$ users & $3 / 2,144$ & $5 / 2,096$ & 0.90 & $\begin{array}{l}0.64 \\
1.26\end{array}$ & Low & Too low \\
\hline $\begin{array}{l}\text { Chang et al., } \\
2011^{* * * * *}[44]\end{array}$ & $\begin{array}{l}\text { Glargine users, } \\
\text { not using int-/ } \\
\text { long-acting } \mathrm{HI}\end{array}$ & $\begin{array}{l}\text { Non-glargine int/long- } \\
\text { acting HI users }\end{array}$ & $6 / 6,558.8^{*}$ & $65 / 47,724.6^{*}$ & 0.53 & $\begin{array}{l}0.21 \\
1.31\end{array}$ & Moderate & Too low \\
\hline $\begin{array}{l}\text { Colhoun et al., 2009a } \\
\text { [5] }\end{array}$ & $\begin{array}{l}\text { Glargine plus } \\
\text { non-glargine } \\
\text { insulin } \\
\text { users }\end{array}$ & $\begin{array}{l}\text { Non-glargine } \\
\text { insulin users }\end{array}$ & O/NR & $29 / 9,667^{*}$ & $\mathrm{NE}$ & NE & High & Too low \\
\hline $\begin{array}{l}\text { Colhoun et al., } \\
2009 b^{* * * * *}[5]\end{array}$ & $\begin{array}{l}\text { Glargine only } \\
\text { users }\end{array}$ & $\begin{array}{l}\text { Non-glargine } \\
\text { insulin users }\end{array}$ & $6 / 1,200^{*}$ & $29 / 9,667^{*}$ & 1.47 & $\begin{array}{l}0.59 \\
3.64\end{array}$ & High & Too low \\
\hline $\begin{array}{l}\text { Currie et al., } \\
2009 b^{* * * * *}[6]\end{array}$ & Glargine users & $\begin{array}{l}\text { Non-glargine } \\
\text { insulin users }\end{array}$ & $10 / 2,245^{*}$ & $38 / 8,102^{*}$ & 0.86 & $\begin{array}{l}0.42 \\
1.75\end{array}$ & Moderate & Too low \\
\hline $\begin{array}{l}\text { Fagot et al., } \\
2013 a^{* * * * *} \text { [47] }\end{array}$ & Glargine users & $\begin{array}{l}\text { Other int-/ } \\
\text { long-acting } \\
\text { insulin only } \\
\text { users }\end{array}$ & $114 / 42,129^{*}$ & $40 / 14,082^{*}$ & 1.08 & $\begin{array}{l}0.72 \\
1.62\end{array}$ & High & Too low \\
\hline $\begin{array}{l}\text { Habel et al., } \\
2013 a^{* * * * *}[51]\end{array}$ & Glargine users & $\mathrm{NPH}$ insulin users & $52 / 10,614.8$ & $217 / 60,868.1$ & 1.3 & $\begin{array}{l}1.0 \\
1.8\end{array}$ & Moderate & Too low \\
\hline $\begin{array}{l}\text { Habel et al., 2013b } \\
\text { [51] }\end{array}$ & $\begin{array}{l}\text { Glargine only } \\
\text { users }\end{array}$ & $\mathrm{NPH}$ insulin users & $33 / 6,402.4$ & $217 / 60,868.1$ & 1.3 & $\begin{array}{l}0.9 \\
2.0\end{array}$ & Moderate & Too low \\
\hline $\begin{array}{l}\text { Habel et al., 2013c } \\
\text { [51] }\end{array}$ & $\begin{array}{l}\text { Glargine and } \\
\mathrm{NPH} \text { insulin } \\
\text { users }\end{array}$ & $\mathrm{NPH}$ insulin users & $19 / 4,212.5$ & $217 / 60,868.1$ & 1.3 & $\begin{array}{l}0.8, \\
2.0\end{array}$ & Moderate & Too low \\
\hline $\begin{array}{l}\text { Kostev et al., } \\
2012 a^{* * * * *} \text { [55] }\end{array}$ & Glargine users & $\mathrm{NPH}$ insulin users & NR & NR & 0.93 & $\begin{array}{l}0.68 \\
1.27\end{array}$ & High & Too low \\
\hline $\begin{array}{l}\text { Lind et al., 2012a***** } \\
\text { [56] }\end{array}$ & Glargine users & $\begin{array}{l}\text { Non-glargine } \\
\text { users }\end{array}$ & $19 / 7,019.4$ & $96 / 48,889.6^{*}$ & 1.54 & $\begin{array}{l}0.90 \\
2.67\end{array}$ & Moderate & Too low \\
\hline $\begin{array}{l}\text { Morden et al., } 2011 \text { a } \\
\text { [59] }\end{array}$ & $\begin{array}{l}\text { Glargine plus } \\
\text { non-glargine } \\
\text { insulin users }\end{array}$ & $\begin{array}{l}\text { Non-glargine } \\
\text { insulin users }\end{array}$ & $102 / 18,889^{*}$ & $333 / 65,294^{*}$ & 1.08 & $\begin{array}{l}0.86 \\
1.36\end{array}$ & High & Low \\
\hline $\begin{array}{l}\text { Morden et al., } \\
2011 b^{* * * * *}[59]\end{array}$ & $\begin{array}{l}\text { Glargine only } \\
\text { users }\end{array}$ & $\begin{array}{l}\text { Non-glargine } \\
\text { insulin users }\end{array}$ & $118 / 21,071^{*}$ & $333 / 65,294^{*}$ & 1.03 & $\begin{array}{l}0.83 \\
1.29\end{array}$ & High & Low \\
\hline $\begin{array}{l}\text { Ruiter et al., } \\
2012 a^{* * * * *}[64]\end{array}$ & $\begin{array}{l}\text { Glargine only } \\
\text { users }\end{array}$ & $\begin{array}{l}\text { Human insulin } \\
\text { only users }\end{array}$ & $11 / 6,875^{*}$ & $N R ; I R=2.28^{*}$ & 1.65 & $\begin{array}{l}1.10 \\
2.47\end{array}$ & Moderate & Too low \\
\hline $\begin{array}{l}\text { Sturmer et al., 2013a } \\
* * * * \\
{[65]}\end{array}$ & Glargine users & $\mathrm{NPH}$ users & $103 / 26,277$ & $19 / 5,885$ & 1.07 & $\begin{array}{l}0.65 \\
1.75\end{array}$ & Moderate & Too low \\
\hline $\begin{array}{l}\text { Suissa et al., } \\
2011 a^{* * * * *}[66]\end{array}$ & Glargine users & $\begin{array}{l}\text { Non-glargine } \\
\text { insulin users }\end{array}$ & $18 / 6,094$ & $60 / 12,262$ & 0.8 & $\begin{array}{l}0.3 \\
2.1\end{array}$ & Moderate & Too low \\
\hline
\end{tabular}


Table 3 Relative risk estimations for breast cancer among insulin treatment groups and the evaluation of bias and power of the studies (Continued)

\begin{tabular}{|c|c|c|c|c|c|c|c|c|}
\hline Pooled hazard ratio & Glargine & No glargine & & & 1.04 & $\begin{array}{l}0.91 \\
1.17\end{array}$ & & \\
\hline \multicolumn{9}{|l|}{$\begin{array}{l}\text { Glargine-no glargine: } \\
\text { incidence rate ratio }\end{array}$} \\
\hline $\begin{array}{l}\text { Ljung et al., 2011a } \\
\text { [57] }\end{array}$ & $\begin{array}{l}\text { Glargine plus } \\
\text { non-glargine } \\
\text { insulin users }\end{array}$ & $\begin{array}{l}\text { Non-glargine } \\
\text { insulin users }\end{array}$ & $59 / 25,033$ & 283/101,419 & 1.04 & $\begin{array}{l}0.77 \\
1.41\end{array}$ & High & Low \\
\hline $\begin{array}{l}\text { Ljung et al., } 2011 \mathrm{~b} \\
{[57]}\end{array}$ & $\begin{array}{l}\text { Glargine only } \\
\text { users }\end{array}$ & $\begin{array}{l}\text { Non-glargine insulin } \\
\text { users }\end{array}$ & $31 / 7,302$ & $283 / 101,419$ & 1.58 & $\begin{array}{l}1.09 \\
2.29\end{array}$ & High & Too low \\
\hline \multicolumn{9}{|l|}{$\begin{array}{l}\text { Glargine-no glargine: } \\
\text { odds ratio }\end{array}$} \\
\hline $\begin{array}{l}\text { Grimaldi-Bensouda } \\
\text { et al., 2013a [49] }\end{array}$ & Glargine users & Non-glargine users & $78 / 287$ & $697 / 2,763^{*}$ & 1.04 & $\begin{array}{l}0.76 \\
1.44\end{array}$ & Low & Borderline \\
\hline $\begin{array}{l}\text { Grimaldi-Bensouda } \\
\text { et al., 2013b [49] }\end{array}$ & Glargine users & $\begin{array}{l}\text { Non-glargine insulin } \\
\text { users }\end{array}$ & $74 / 203$ & $70 / 207$ & 0.96 & $\begin{array}{l}0.61 \\
1.53\end{array}$ & Low & Too low \\
\hline $\begin{array}{l}\text { Grimaldi-Bensouda } \\
\text { et al., 2013c [49] }\end{array}$ & Glargine users & $\begin{array}{l}\text { Human } \\
\text { insulin users }\end{array}$ & $N R$ & $N R$ & 1.29 & $\begin{array}{l}0.78 \\
2.13\end{array}$ & Low & $\mathrm{NE}$ \\
\hline $\begin{array}{l}\text { Grimaldi-Bensouda } \\
\text { et al., 2013d [49] }\end{array}$ & Glargine users & Aspart users & $N R$ & $N R$ & 1.10 & $\begin{array}{l}0.64 \\
1.89\end{array}$ & Low & $N E$ \\
\hline $\begin{array}{l}\text { Grimaldi-Bensouda } \\
\text { et al., 2013e [49] }\end{array}$ & Glargine users & Lispro users & $N R$ & $N R$ & 0.85 & $\begin{array}{l}0.48 \\
1.50\end{array}$ & Low & $N E$ \\
\hline $\begin{array}{l}\text { Mannucci et al., } \\
\text { 2010a [58] }\end{array}$ & Glargine users & $\begin{array}{l}\text { Non-glargine } \\
\text { insulin users }\end{array}$ & $N R$ & $N R$ & $N E$ & $\mathrm{NE}$ & High & Too low \\
\hline \multicolumn{9}{|l|}{$\begin{array}{l}\text { Determir-no } \\
\text { determir: hazard } \\
\text { ratio }\end{array}$} \\
\hline $\begin{array}{l}\text { Fagot et al., 2013b } \\
\text { [47] }\end{array}$ & Determir users & $\begin{array}{l}\text { Other int-/ } \\
\text { long-acting } \\
\text { insulin only users }\end{array}$ & $38 / 12,806^{*}$ & $116 / 43,131^{*}$ & 1.08 & $\begin{array}{l}0.72 \\
1.62\end{array}$ & High & Too low \\
\hline $\begin{array}{l}\text { Kostev et al., 2012b } \\
\text { [55] }\end{array}$ & Detemir users & NPH insulin users & NR/789 & $N R / 4,206$ & 1.17 & $\begin{array}{l}0.66 \\
2.06\end{array}$ & High & Too low \\
\hline \multicolumn{9}{|l|}{$\begin{array}{l}\text { Determir-no } \\
\text { determir: incidence } \\
\text { rate ratio }\end{array}$} \\
\hline $\begin{array}{l}\text { Dejgaard et al., 2009a } \\
\text { [46] }\end{array}$ & Determir users & $\mathrm{NPH}$ users & $1 / 2,252$ & $0 / 1,420$ & $\mathrm{NE}$ & $\mathrm{NE}$ & Low & Too low \\
\hline $\begin{array}{l}\text { Dejgaard et al., 2009b } \\
\text { [46] }\end{array}$ & Determir users & Glargine users & $1 / 917$ & $3 / 628$ & $N R$ & NR & Low & Too low \\
\hline \multicolumn{9}{|l|}{$\begin{array}{l}\text { Aspart-no aspart: } \\
\text { odds ratio }\end{array}$} \\
\hline $\begin{array}{l}\text { Grimaldi-Bensouda } \\
\text { et al., 2013f [55] }\end{array}$ & Aspart users & Non-aspart users & $54 / 241$ & $721 / 2,809^{*}$ & 0.95 & $\begin{array}{l}0.64 \\
1.40\end{array}$ & Low & Borderline \\
\hline
\end{tabular}


Table 3 Relative risk estimations for breast cancer among insulin treatment groups and the evaluation of bias and power of the studies (Continued)

Lispro-no lispro: odds ratio

\begin{tabular}{|c|c|c|c|c|c|c|c|c|}
\hline $\begin{array}{l}\text { Grimaldi-Bensouda } \\
\text { et al., 2013g [49] }\end{array}$ & Lispro users & Non-lispro users & $46 / 133$ & $729 / 2,917^{*}$ & 1.23 & $\begin{array}{l}0.79 \\
1.92\end{array}$ & Low & Borderline \\
\hline \multicolumn{9}{|l|}{$\begin{array}{l}\text { Human insulin-no } \\
\text { human insulin: } \\
\text { hazard ratio }\end{array}$} \\
\hline $\begin{array}{l}\text { Fagot et al., 2013c } \\
\text { [47] }\end{array}$ & $\begin{array}{l}\text { Basal human } \\
\text { insulin users }\end{array}$ & $\begin{array}{l}\text { Other int-/ } \\
\text { long-acting } \\
\text { insulin only } \\
\text { users }\end{array}$ & $15 / 5,813^{*}$ & $139 / 50,948^{*}$ & 1.03 & $\begin{array}{l}0.56 \\
1.88\end{array}$ & High & Too low \\
\hline Gu et al., 2013 [50] & $\begin{array}{l}\text { Human insulin } \\
\text { users }\end{array}$ & No insulin users & $4 / 6,188^{*}$ & $14 / 10,435^{*}$ & 0.33 & $\begin{array}{l}0.10 \\
1.13\end{array}$ & Moderate & Too low \\
\hline $\begin{array}{l}\text { Ruiter et al., 2012b } \\
\text { [64] }\end{array}$ & $\begin{array}{l}\text { Non-glargine } \\
\text { insulin users }\end{array}$ & $\begin{array}{l}\text { Human insulin } \\
\text { only users }\end{array}$ & $31 / 15,578^{*}$ & $N R ; I R=2.28^{*}$ & 0.99 & $\begin{array}{l}0.81 \\
1.20\end{array}$ & Moderate & Too low \\
\hline \multicolumn{9}{|l|}{$\begin{array}{l}\text { Human insulin-no } \\
\text { human insulin: } \\
\text { odds ratio }\end{array}$} \\
\hline $\begin{array}{l}\text { Grimaldi-Bensouda } \\
\text { et al., 2013h [49] }\end{array}$ & Human insulin users & $\begin{array}{l}\text { Non-human } \\
\text { insulin users }\end{array}$ & $59 / 260$ & $716 / 2,790^{*}$ & 0.81 & $\begin{array}{l}0.55 \\
1.20\end{array}$ & Low & Borderline \\
\hline
\end{tabular}

Bold values are significantly different * ${ }^{*}$ Calculated using data provided (if not indicated directly these were taken from the table in the paper) **Risk estimates are adjusted for covariates as stated in Additional file 1 : Table S3. Covariates used in the various analyses are the same within one study. ${ }^{* *}$ Case-control studies. ${ }^{* * *}$ Cohort studies or randomized clinical trials. ${ }^{* * * *}$ Included in meta-analysis. ${ }^{* * * * *}$ The exposure of interest is the exposure comparison group in this analysis. Studies are first ordered by type of exposure and then by type of risk estimate. Note: Hiesh 2012 is a cohort study but provided odds ratio estimates in the paper.

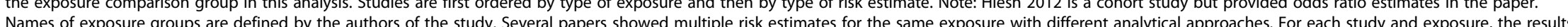
from the lest bias or be from the least biased or best pefformed analyses are shown; showing hazard ratios, incidence rate ratios or odds ratios as applicable. Different exposure comparisons within one study are indicated by a,b,c etc. We choose to include the isk estimate that gave (in order of importance). 1) estimates for incident users was preferred over estimates for prevalent users, 2) as-teated analysis (during study period/follow up) was prefered ove inention estimate. NR not reported, NE not estimated, HI human insulin, TZD Thiazolidinedione, NIAD non-insulin anti-diabetic drug, NPH Neutral Protamine Hagedorn, Int intermediate. 


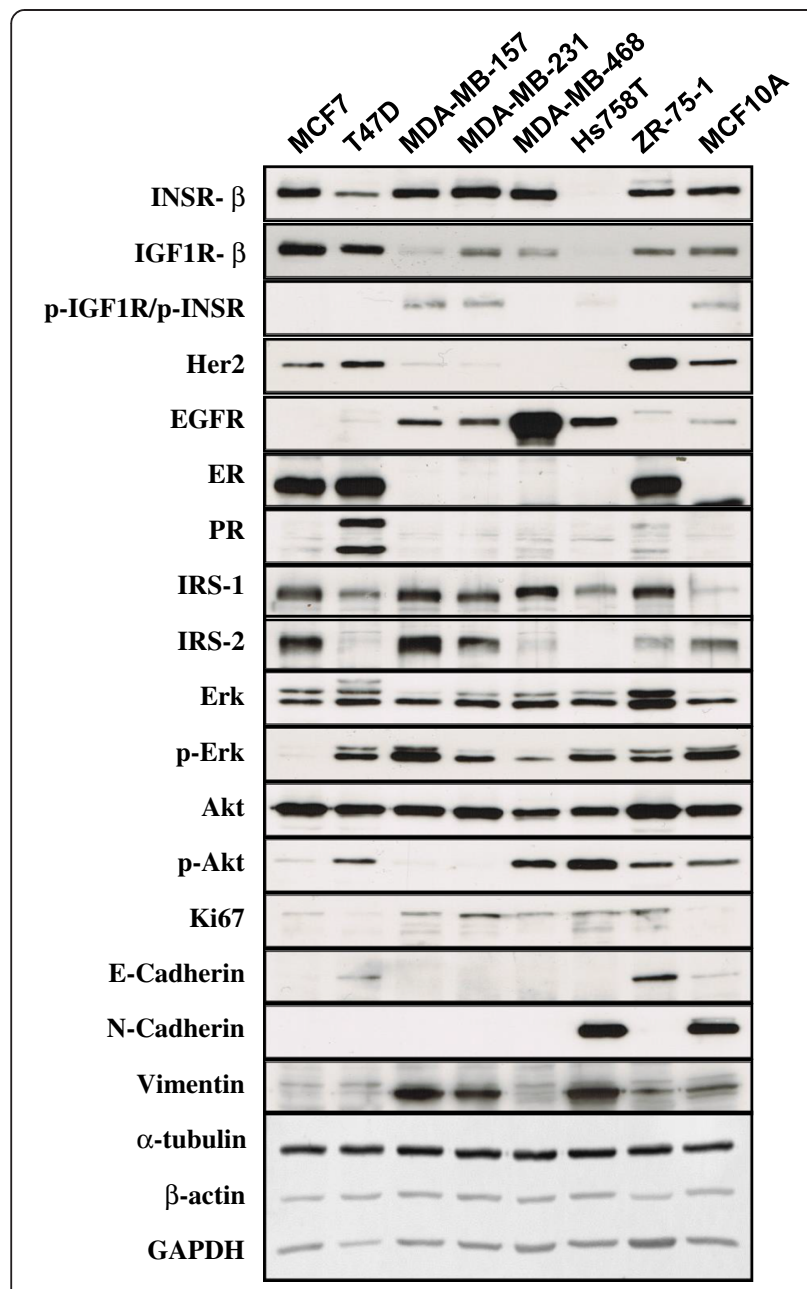

Fig. 2 Protein expression profiling of eight commonly used human breast cell lines. Receptor levels and signalling molecules downstream of the insulin receptor/insulin-like growth factor-1 receptor (INSR/IGF1R) signalling pathway have been quantified. Furthermore some breast cancer subtype markers have been used to further characterize these cell lines that are commonly used in the research articles discussed in this review. Her2 human epidermal growth factor receptor 2, EGFR epidermal growth factor receptor, ER oestrogen receptor, $P R$ progesterone receptor, Erk extracellular signal-related kinase, GAPDH glyceraldehyde-3-phosphate dehydrogenase IRS Insulin Receptor Substrate

upregulated [28, 31]. The clinical relevance of this increased mitogenic potential is as yet unknown because glargine is rapidly metabolised in vivo into two metabolically active compounds, M1 and M2 $[69,70]$. These metabolites possess low mitogenic signalling $[28,34]$.

In a 2-year follow up study, wild-type Sprague-Dawley rats, Wistar rats, and NMRI mice have been used to test the effect of chronic glargine injections compared to the insulin NPH injections; no difference in tumour-free survival was observed [37, 38] (Table 2). In contrast, a recent study revealed a (non-significant) decrease in tumour latency time after a similar chronic exposure to glargine; tumour multiplicity or metastases were not affected [40]. Glargine injections induced no increased receptor activation response in the mammary glands of Sprague-Dawley rats [39].

Three randomized clinical trials (RCT) that investigated breast cancer risk among glargine users compared to non-glargine users $[42,52,63]$ did not show significant differences (Table 3). Most case-control and cohort studies showed a non-significant increased risk. Only two observational studies [57, 64] showed a statistically significant increased risk of breast cancer with an IRR of 1.58 (95\% CI 1.09, 2.29) and HR of 1.65 (95 \% CI 1.10, $2.47)$, respectively. Both studies included glargine-only users and compared them to non-glargine insulin users [57] and human-insulin-only users [64]. As the glargine studies did not show statistically significant heterogeneity $\left(I^{2}=0.0 \% ; \mathrm{p}>0.05\right)$ a meta-analysis was performed. From 13 studies the pooled HR for glargine vs no use of glargine was 1.04 (95\% CI 0.91, 1.17; $p=0.49$ ) (Fig. 3 and Table 3), showing no evidence for an association between insulin glargine treatment and increased incidence of breast cancer.

\section{Insulin detemir}

Like glargine, detemir is a long-acting insulin analogue. In general, it is assumed that detemir has a lower mitogenic potential compared to human insulin [22, 28, 31, 34], but in a number of in vitro studies similar proliferation or even increased proliferation of determir has been observed $[25,29,35]$ (Table 1). The binding characteristics of detemir to albumin are different among species. In almost all in vitro studies BSA or FBS is added to the stimulation medium. Interpretation of these mitogenicity studies is difficult because it is not yet known how the bovine albumin interacts with detemir compared to human albumin [11]. For the same reason it is not surprising that no chronic animal studies have been conducted with insulin detemir. Only three epidemiological studies have been performed, one RCT [46] and two cohort studies [47, 55]; none found an association with breast cancer development (Table 3).

\section{Insulin aspart, glulisine and lispro}

Compared to glargine and detemir, the insulin analogues aspart, glulisine and lispro are less well evaluated for mitogenic potential; no increased mitogenic action was found in four in vitro studies [25, 28, 30, 34] (Table 1). Only one in vitro study suggested a small non-significant proliferative increase of aspart compared to human insulin [31]. Another in vitro study found the mitogenic potential of glulisine to be significantly lower than human insulin [30]. Evidence that lispro and glulisine had increased proliferative potential 


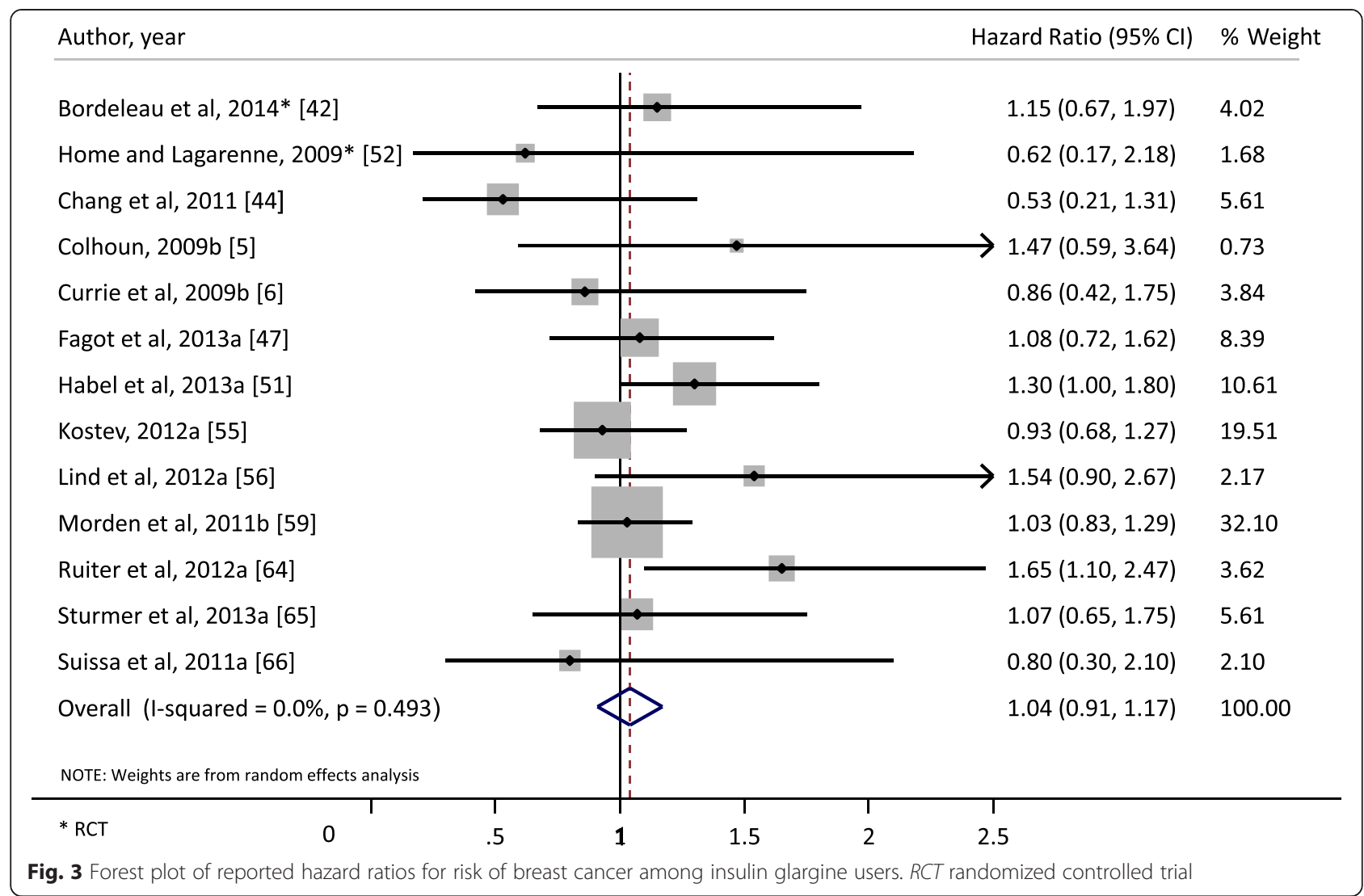

was found in just one in vitro study and for just two of the tested cell lines (MDA-MB-157 and MDA-MB-468) [29]. We previously found that the PI3K signalling cascade is significantly more upregulated after lispro treatment than human insulin stimulation only in the IGF1R overexpressing MCF7 cell line [34]. Similar to the in vitro data, the epidemiological data on these short-acting insulin analogues is scarce. Just one study reports ORs for aspart and lispro of 0.95 (95\% CI 0.64, 1.40 ) and 1.23 (95\% CI $0.79,1.92)$, respectively [49] (Table 3).

\section{Human insulin}

In vitro studies showed that treatment of diabetics with human insulin has low mitogenic potential (Table 1). From the in vivo studies it can be concluded that human insulin is not carcinogenic as the number of tumours that developed in the human-insulin-treated group was similar to the vehicle-injected group (Table 2). Only three epidemiological studies explored the effect of human insulin as the exposure of interest on the risk of breast cancer. Two of these studies compared human insulin users with insulin analogue users [47, 49] and found no significant difference in breast cancer risk (Table 3). The other study compared human insulin users with diabetics not treated with insulin and reported a HR of 0.33 with a relatively wide $95 \% \mathrm{CI}$ of $0.10,1.13$ [50]. This study was under powered.

Human insulin, especially NPH, was often used as exposure comparison group in the studies that investigated risk of breast cancer related to insulin analogue use. Most of these studies did not report significant differences in the risk of breast cancer, as mentioned previously.

\section{Insulin AspB10}

The increased carcinogenic effect of insulin AspB10 had already been discovered in 1992 [71]. Since then this insulin analogue has been used in many in vitro studies as a reference compound with a strong carcinogenic potential. In proliferation studies AspB10 was highly mitogenic compared to human insulin, irrespective of the cell line used [21, 22, 26, 27, 29, 34] (Table 1). Most studies indicated that AspB10 induces proliferation by increased IGF1R signalling, but there are indications that the INSR is also involved because increased proliferation was not fully blocked when using a specific IGF1R inhibitor [26]. One study used two murine mammary tumour cell lines, both expressing INSR and IGF1R. These cell lines were stimulated with AspB10 and only activation of INSR and not IGF1R was observed [20]. In 
a different study it was indicated that a prolonged occupancy time of this analogue towards the INSR results in sustained activation of this receptor and subsequently increased mitogenic potency [22]. With a collagen invasion assay it was determined in several breast cancer cell lines that AspB10 has an increased invasive capacity compared to human insulin [29]. In a very elaborate kinase/inhibitor study it was found that multiple core kinases are involved in the mitogenic action of AspB10, because phosphorylation of AKT, p70S6K, S6, and 4EBP1 was found to be increased compared to human insulin exposure [27].

In animal studies, AspB10 was found to have dosedependent increased carcinogenic potential [71] (Table 2). Xenograft rodent models with injected mammary gland tumour cell lines were treated with either human insulin or AspB10. Tumours were significantly bigger after the AspB10 injections and, although not significant, more lung metastases were found in this treatment group. Strong upregulation of p-AKT has been observed on kinase activation analysis of these tumours, indicating that the carcinogenic effects of AspB10 might be a direct effect from a PI3K response [20]. A very recent study used a p53 ${ }^{\mathrm{R} 270 \mathrm{H} /+}$ WAPCre mouse model, which develops spontaneous human relevant mammary gland tumours within 70 weeks, to show that chronic exposure to AspB10 significantly decreases the tumour latency time. A detailed protein expression analysis showed that tumours induced by AspB10 or IGF1 have a distinct expression pattern compared to tumours from insulin- or vehicle-treated mice; both the PI3K and the MAPK were found to be significantly upregulated after AspB10 and IGF1 treatment [40]. A different study focussed on the short term mitogenic effects of AspB10 and found significantly stronger receptor activation in the mammary glands of SpragueDawley rats one hour after AspB10 injections compared to human insulin treatment [39]. As insulin AspB10 has been shown to have mitogenic properties in in vitro and animal studies, this drug has never been available to humans.

\section{Insulin (analogue) users versus non-insulin users or NIAD users}

In the epidemiological studies, the risk of breast cancer mostly showed non-significant decreased associations with insulin use vs non-insulin use (drug exposure undefined) (Table 3). These studies did not distinguish between insulin analogues and human insulin. In contrast, most studies that compared insulin users with NIAD users (irrespective of the type of NIAD used) showed non-significant increased associations with risk of breast cancer. Only one study comparing insulin users versus non-insulin-users showed a statistically significant decreased risk of breast cancer (HR 0.86; 95 \% CI 0.81,
0.91) in patients with type 2 diabetes [60]. However, we judge this study to be biased because the risk estimates were not adjusted for important risk factors for breast cancer and DM, immortal time bias might be present, and no data on duration of exposure were available. Exposure categories (insulin use-no insulin use and insulin use-NIAD only use) are hard to define and compare, because many patients with type 2 diabetes are using insulin (analogues) simultaneous with NIADs. Most studies that are included in this review investigated combined categories of exposure to insulin (analogues) and NIADs.

\section{Dose and duration effects in epidemiological studies}

No significant differences were found between strata of duration and risk of breast 5 five years of any insulin treatment (HR 2.25; $95 \%$ CI 0.72, 6.99) [62]. Among the glargine users, the study with the longest follow up comparing exposure of 4-7 years versus $<4$ years did not observe increased breast cancer risk [49]. Another study revealed that the risk of breast cancer increased in the first 3 years after the start of insulin glargine use, after which the risk of breast cancer remained at the same level [56]. Results of the effect of glargine dose on the occurrence of breast cancer $[47,49,56,58,59,64]$ produced inconsistent results (Additional file 1: Table S4). Some studies found significantly increased relative risks with increasing dose $[56,59,64]$, while others did not $[47,49,58,59]$; this seems partly dependent on the exposure definition. Only one of the studies investigating glargine dose used cumulative dose [47]. The results of one in vivo study in humans indicated that there is almost no glargine circulating in plasma regardless of the dose given. Plasma M1 concentration increased with increasing dose of glargine, but as was mentioned previously, M1 possesses low mitogenic signalling [70].

\section{Discussion}

Limitations of the studies and interpretation of the findings

\section{In vitro studies}

The large variation in published in vitro results can be explained by differences in study design. For example, the choice of cell line greatly affects the obtained results because the responsiveness to growth factors, like insulin and insulin analogues, may be different from one cell line to another. Based on the cell line characterisation (Additional file 1: Table S1), we showed there is a striking variation in receptor expression of the human cell lines used.

Different cell lines also have different expression of the relevant receptors involved in the insulin response. The MDA-MB-231 cell line has very low expression of IGF1R. Therefore, the increased mitogenic potential of 
glargine (due to enhanced IGF1R signalling) could not be detected in this cell line [28]. However, using the MCF7 cell line (which expresses very high levels of IGF1R) the increased mitogenic potential of this compound became evident [28]. Other cell lines with low or moderate expression levels of IGF1R are less suitable for a mitogenic evaluation of insulin analogues. In line with this, a recent study including four different breast cancer cell lines (MCF7, MDA-MB-157, MDA-MB-468 and T47D) found that mitogenicity of growth factors strongly depends on the cell line that was used [29]. However, the authors concluded that the INSR/IGF1R status was not the only explanatory factor. Therefore, we determined the expression of downstream signalling molecules (Fig 2). This illustrated that the poor responsiveness in the T47D and MDA-MB-468 cell lines upon glargine exposure $[25,29]$ may be explained by low expression of IRS1 (T47D) or IRS2 (MDA-MB-468), the first downstream targets of the INSR/IGF1R.

Besides INSR/IGF1R signalling other receptors also might have a role in insulin (analogue)-induced mitogenicity. Due to insulin-oestrogen receptor/progesterone receptor (ER/PR) crosstalk the IRS1 and subsequently the PI3K and MAPK signalling cascades can be upregulated resulting in enhanced proliferation [72]. This effect might contribute to the increased insulin (analogue) sensitivity of MCF7, T47D and ZR-75-1 compared to the triple-negative cell lines (MDA-MB-157, MDA-MB-231, MDA-MB-468 and MCF10A). Therefore, it is important to point out that primarily ER-positive or triple-negative breast cancer cell lines have been used in the included studies.

The majority of the mitogenicity studies used the MCF7 cell line [23-35]. It is desirable that future studies include different cell lines, so that cell-line-specific effects can be excluded. For translational reasons it is essential that protein expression (and especially receptor profiles) in benign human mammary gland tissues are quantified, only in that way we can determine which cell model has the highest clinical relevance.

Another important quality factor is the starvation method. For a proper effect of a specific stimulation it is essential that the target cells are deprived from other growth factors. Some studies did not starve their cells prior to the start of the assay [21, 25, 28,33]; for shortterm assays especially, this might have major consequences. Finally, the use of proper positive and negative controls is most important for a good quality experiment. Some studies [32, 33] did not include a positive control while others lack a negative control [23], thereby making it impossible to put the results in perspective. Furthermore, one study did include a positive control (IGF1) [24], but this compound did not show a positive effect, which questions the sensitivity of their experiments.

\section{Animal studies}

The type of animal model used plays a major role in the quality of animal studies. Generally, it is thought that rats are more sensitive in terms of carcinogenicity towards compounds and have a higher clinical relevance than mouse models [73]. But there are also major disadvantages, like higher costs and the lack of good humanized breast cancer rat models. Two studies that used rats have rather small group sizes, which obviously affects the power of their studies [37-39]. The doses that were used in the reviewed animal studies are quite comparable to each other and are all thought to be supraphysiological (i.e., over 50 times the human dose, based on $\mathrm{nmol} / \mathrm{kg}$ ). In one study a non-equimolar comparison was made between the different compounds, but doses had been chosen to induce an equi-pharmacological/ metabolic response [40]. High mortality was observed in another study, probably due to hypoglycaemia, therefore the dose was lowered in a later phase of this study [39]. Surprisingly, other studies that used similar doses did not observe hypoglycaemia $[37,38,40]$. To verify the sensitivity of the models and techniques it is essential that the appropriate controls are included. Half of the included animal studies lacked proper controls. In our opinion both insulin and IGF1 (and ideally also AspB10) should always serve as controls to be able to put the obtained results into perspective.

\section{Epidemiological studies}

The epidemiological studies included in this review have many limitations and results are difficult to compare across studies because the exposure of interest and exposure comparison groups have been defined differently. For example, some studies compared glargine-only users with human-insulin-only users [64], while others compared glargine users with non-glargine-insulin users [66]. In this case, the comparator is a mix of several exposures, which may affect the conclusion about the effect of certain insulins (analogues). Some studies examined several definitions for the exposure of interest and indeed this resulted in slightly different effect estimates $[57,59]$. Moreover, it is difficult to disentangle the effect of insulin and the role of NIADs because most diabetic patients treated with insulin have prescriptions of NIADs as well. However, it is important to do so, because some studies have shown anti-tumour effects of metformin, the most prescribed NIAD among patients with type 2 diabetes [74]. Of note, the quality of some of these metformin studies is doubtful as well.

Inclusion criteria differed largely among studies. For example, some studies included patients with only one insulin prescription while others included continuous users over a period of 6 months. More important, there was large variation in the definition of time of exposure. 
Some studies determined the use of different insulin types at baseline or during a fixed period (intention to treat), while others determined insulin exposure during follow up (time-dependently). This may lead to patients with only one specific insulin prescription during follow up being falsely classified as continuous users during the whole period. Cumulative exposure over time, censoring for discontinuation, or switching and latency period could affect the results. The uncertainty surrounding the extent to which a registered prescription dispensed for an insulin analogue reflects real life use of insulin analogues limits the ability to detect the true effect on the occurrence of breast cancer. Furthermore, studies variably included incident and prevalent users of insulin compromising estimates of association between the duration of use and breast cancer development.

Other methodological aspects that are important when interpreting the results of these studies are incorrect and too short an exposure time (maximum 3.8 years mean exposure time), reverse causation, confounding by indication, and residual confounding (Additional file 1: ESM $3)$. Most studies were based on type $2 \mathrm{DM}$, and/or did not specify the type of DM. Risk of bias was classified as low (for definition see Additional file 1: ESM 3) in only five studies [42, 46, 49, 62, 63], but the power of these studies was inadequate (Additional file 1: Table S5). Of these studies, only two considered breast cancer as a main outcome $[49,62]$. Most risk estimates have wide CIs, due to lack of power of the study. Two of the three studies that found significantly different results were classified as having a high risk of bias $[57,60]$ or had lack of power [57, 64]. So far there is not a single very well-designed study to have investigated insulin treatment and breast cancer risk as the main outcome, with sufficient power. The included RCTs had limitations too, such as limited follow up (except for one RCT with a follow up of 6 years [42]), insufficient power, or cancer incidence as a secondary outcome $[63,75]$.

\section{All layers of evidence in perspective}

Studies in humans are the gold standard for evaluating evidence of exposure and disease. The epidemiological studies reviewed varied in study design and exposure definition to too large an extent among different insulin analogues to evaluate their impact on breast cancer risk estimates. The risk estimates seemed not to be biased by important confounders, as adjusted and unadjusted risk estimates only differed slightly. However, unmeasured confounding may still be present. In addition, the upper limit of the $95 \% \mathrm{CI}$ of the pooled risk estimate of breast cancer among glargine users was 1.17. This strengthens our idea that if any, the increased risk of breast cancer due to currently used insulin (analogues) is likely to be very small.
A distinction should be made between studying tumour initiation or progression, though in the human setting it difficult to discern these because of potential lag time in the detection of cancer. The epidemiological studies investigated the incidence of primary breast tumours upon insulin treatment in DM patients. True tumour initiation in animal studies can only be investigated with long-term exposure in rodents, which are costly experiments. The animal xenograft models and in vitro studies of mammary tumour cell lines summarized here investigated tumour progression; e.g., by evaluation of cell proliferation or upregulation of mitogenic pathways. All together, the results of this systematic review suggest that insulin treatment might be involved in tumour promotion.

Another issue to be raised is that breast cancer is not one disease but consists of different subtypes, e.g., ERpositive or ER-negative cancer with different prognoses. The promotion of tumour cell growth upon insulin exposure may differ for different breast cancer subtypes. However, there are very limited human/epidemiological data from only two studies on the association of tumour subtypes and insulin (analogues) exposure among diabetic patients with breast cancer $[49,76]$. More data are available on the prognosis of diabetic patients with breast cancer. It has been shown that overall mortality after breast cancer diagnosis is 25 to 50 \% higher in diabetic women compared to their non-diabetic counterparts $[45,62,77,78]$, even after adjustment for tumour stage $[77,78]$. However, whether this increased mortality is breast cancer-related or caused by comorbidities related to DM is not clear. Breast cancer in patients with $\mathrm{DM}$ is often diagnosed at an advanced stage compared to patients without DM [77-80]. But studies that investigated the association between breast cancer-specific mortality and diabetes have inconsistent results $[45,78,80,81]$. Among patients with type $2 \mathrm{DM}$, insulin treatment is associated with a worse cancer outcome and increased all-cause mortality compared to metformin treatment [78, 82]. Only one study investigated the effect of cumulative dose and duration of insulin treatment on breast-cancer-specific survival, and found lower mortality from breast cancer [83].

\section{Conclusion}

Based on the current epidemiological and animal data there is no compelling evidence that any clinically available insulin analogue, or human insulin increases breast cancer risk. However, animal data were limited and there is not a single very well-designed epidemiological study to have investigated insulin treatment and breast cancer risk as the main outcome, and with sufficient power. Large randomized clinical trials were negative for increased breast cancer risk with glargine, but longer 
follow up may be needed to detect delayed or smaller effects. In vitro studies have shown that only insulin AspB10 and glargine have increased mitogenic potential compared to regular human insulin in breast cancer cell lines. The relevance of this finding for the clinical situation is unknown because AspB10 is not used in humans and it has been shown that glargine is rapidly metabolized in vivo into M1 and M2, metabolites with low mitogenic potential. Evidence on the potential pathways involved in insulinanalogue-induced breast cancer mitogenesis is limited.

\section{Unanswered questions and future research}

Except for insulin AspB10, which has never been available to humans, all insulin analogues are still marketed. Although, there is evidence from in vitro data that insulin glargine has increased mitogenic potential, so far, epidemiological studies have not shown evidence for an association between insulin (analogue) treatment and breast cancer risk in female diabetic patients. However, due to a relatively short follow up time in the epidemiological studies, it cannot be excluded that diabetic patients with pre-neoplastic lesions might be at higher risk of developing an invasive tumour when given a specific insulin treatment. Research on this topic is important but is still largely lacking. Therefore, we are awaiting the results of ongoing efforts to pool multiple large national databases from different countries to perform a retrospective observational study in humans with a proper design, enough patients and long follow up. Additionally, further research into the aetiology of insulin and breast cancer development is important.

\section{Additional file}

Additional file 1: ESM 1. Search strategy for each database, study selection and results. ESM 2. Characterization of cells lines. Additional materials and methods section. ESM 3. Method for quality evaluation of epidemiological studies. ESM 4. Description of the included studies. Table S1. Protein and gene expression of hormone receptors for in vitro human mammary cell lines included. Table S2. Description of epidemiological studies included in the systematic review. Table S3. a Characteristics of the case control studies included in the systematic review. $\mathbf{b}$ Characteristics of the cohort studies included in the systematic review. c Characteristics of the randomized clinical trials included in the systematic review. Table S4. Relative risk estimations for breast cancer among different duration and dose categories within insulin treatment groups. Table S5. Quality evaluation of the epidemiological studies included in the systematic review*. Figure S1. Forest plot of breast cancer risk among insulin (analogue) users stratified by treatment group and type of effect estimate. Different exposure comparisons within one study are indicated by $a, b, c$. The exposure comparison can be found in Table 3 and Additional file 1: Table S2. (PDF $457 \mathrm{~kb}$ )

\section{Abbreviations}

AKT: protein kinase B; BrdU: 5-Bromo-2'-deoxyuridine; BSA: bovine serum albumin; DM: diabetes mellitus; DMEM: Dulbecco's modified Eagle's medium; EGFR: epidermal growth factor receptor; EMT: Epithelial to mesenchymal Transition; ER: oestrogen receptor; ERK: extracellular signal regulated kinase; FACS: fluorescence-activated cell sorting; FBS: foetal bovine serum;
FCS: foetal calf serum; GAPDH: glyceraldehyde-3-phosphate dehydrogenase; HER2: human epidermal growth factor receptor 2; HI: human insulin; HR: hazard ratio; IGF1R: insulin-like growth factor 1 receptor; INSR: insulin receptor; IRR: incidence rate ratio; IU: international unit; MAPK: mitogen activated protein kinase; mTOR: mechanistic target of rapamycin; NIAD: noninsulin anti-diabetic drug; OR: odds ratio; PI3K: phosphatidylinositol-4,5bisphosphate 3-kinase; PR: progesterone receptor; PRISMA: preferred reporting items for systematic reviews and meta-analyses; PTEN: phosphatase and tensin homolog; RCT: randomized controlled trial; RR: relative risk; Sig: significance; TZD: thiazolidinedione; WB: Western Blot.

\section{Competing interest}

The authors declare that there is no duality of interest associated with this manuscript.

\section{Authors' contributions}

MKS, JWVDL, CLES, BVDW and ADB conceived this study. HB and BTB extracted and analysed the data. BTB performed the protein quantification experiments on the cell lines. $\mathrm{HB}$ and $\varnothing \mathrm{K}$ evaluated the quality of the epidemiological studies. HB, BTB, MKS and CLES interpreted the data and wrote the manuscript. All authors critically revised the manuscript for important intellectual content and approved the final version of the manuscript.

\section{Acknowledgements}

We acknowledge the Sanger institute for providing the receptor gene expression levels of the cell lines. The research leading to the results of this study has received funding from the European Community's Seventh Framework Programme (FP-7) under grant agreement number 282526, the CARING project. The funding source had no role in study design, data collection, data analysis, data interpretation or writing of the report. MKS was funded by the Dutch Cancer Society project number DCS-NKI2009-4363. BTB was funded by the SOR project 360003 from the National Institute for Public Health and the Environment (RIVM), Bilthoven, the Netherlands.

\section{Author details}

'Division of Molecular Pathology, The Netherlands Cancer Institute, Plesmanlaan 121, 1066 CX Amsterdam, The Netherlands. ${ }^{2}$ Division of Toxicology, Leiden Academic Centre for Drug Research, Leiden University, Leiden, The Netherlands. ${ }^{3}$ Department of Pharmacoepidemiology, Norwegian Institute of Public Health, Oslo, Norway. ${ }^{4}$ Departments of Clinical Medicine and Endocrinology, Aalborg University, Aalborg, Denmark. ${ }^{5}$ Department of Endocrinology and Internal Medicine (MEA), Aarhus University Hospital THG, Aarhus, Denmark. ${ }^{6}$ Division of

Pharmacoepidemiology \& Clinical Pharmacology, Utrecht Institute for Pharmaceutical Sciences, Utrecht University, Utrecht, Netherlands. ${ }^{7}$ Medicines Evaluation Board (MEB), Utrecht, The Netherlands. ${ }^{8}$ Centre for Health Protection, National Institute for Public Health and the Environment (RIVM), Bilthoven, The Netherlands.

Received: 27 February 2015 Accepted: 7 July 2015

Published online: 05 August 2015

\section{References}

1. IARC. Breast cancer fact sheet. 2012.

2. Larsson SC, Mantzoros CS, Wolk A. Diabetes mellitus and risk of breast cancer: a meta-analysis. Int J Cancer. 2007;121:856-62.

3. Home P. Insulin therapy and cancer. Diabetes Care. 2013;36:S240-244.

4. Butler PC. Insulin glargine controversy: a tribute to the editorial team at Diabetologia. Diabetes. 2009;58:2427-8.

5. Colhoun HM. Use of insulin glargine and cancer incidence in Scotland: A study from the Scottish Diabetes Research Network Epidemiology Group. Diabetologia. 2009;52:1755-65.

6. Currie CJ, Poole CD, Gale EA. The influence of glucose-lowering therapies on cancer risk in type 2 diabetes. Diabetologia. 2009;52:1766-77.

7. Hemkens LG, Grouven U, Bender R, Gunster C, Gutschmidt S, Selke GW, et al. Risk of malignancies in patients with diabetes treated with human insulin or insulin analogues: a cohort study. Diabetologia. 2009;52:1732-44.

8. Jonasson JM, Ljung R, Talback M, Haglund B, Gudbjornsdottir S, Steineck G. Insulin glargine use and short-term incidence of malignancies-a populationbased follow-up study in Sweden. Diabetologia. 2009;52:1745-54. 
9. Mollentze WF. A possible link between insulin glargine and malignancy: the facts. Cardiovasc J Afr. 2009;20:216-8. discussion 218-219.

10. Ciaraldi TP, Sasaoka T. Review on the in vitro interaction of insulin glargine with the insulin/insulin-like growth factor system: potential implications for metabolic and mitogenic activities. Horm Metab Res. 2011:43:1-10.

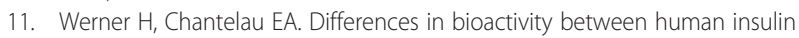
and insulin analogues approved for therapeutic use- compilation of reports from the past 20 years. Diabetol Metabolic Syndrome. 2011;3.

12. Janghorbani M, Dehghani M, Salehi-Marzijarani M. Systematic review and meta-analysis of insulin therapy and risk of cancer. Horm Cancer. 2012:3:137-46.

13. Karlstad O, Starup-Linde J, Vestergaard P, Hjellvik V, Bazelier MT, Schmidt $M K$, et al. Use of insulin and insulin analogs and risk of cancer - systematic review and meta-analysis of observational studies. Curr Drug Saf. 2013;8:333-48.

14. Colmers IN, Bowker SL, Tjosvold LA, Johnson JA. Insulin use and cancer risk in patients with type 2 diabetes: a systematic review and meta-analysis of observational studies. Diabetes Metab. 2012;38:485-506.

15. Du X, Zhang R, Xue Y, Li D, Cai J, Zhou S, et al. Insulin glargine and risk of cancer: a meta-analysis. Int J Biol Markers. 2012;27:e241-246.

16. Tang $X$, Yang L, He Z, Liu J. Insulin glargine and cancer risk in patients with diabetes: a meta-analysis. PLoS One. 2012;7, e51814.

17. PROSPERO: http://www.crd.york.ac.uk/PROSPERO/.

18. Moher D, Liberati A, Tetzlaff J, Altman DG. Preferred reporting items for systematic reviews and meta-analyses: the PRISMA statement. PLoS Med. 2009;6, e1000097.

19. The Cochrane Collaboration. Higgins JPT GS, eds.: Cochrane Handbook for Systematic Reviews of Interventions, Version 5.1.0 [updated March 2011]. 2011.

20. Gallagher EJ, Alikhani N, Tobin-Hess A, Blank J, Buffin NJ, Zelenko Z, et al. Insulin receptor phosphorylation by endogenous insulin or the insulin analog AspB10 promotes mammary tumor growth independent of the IGF-I receptor. Diabetes. 2013;62:3553-60.

21. Glendorf T, Knudsen L, Stidsen CE, Hansen BF, Hegelund AC, Sorensen AR, et al. Systematic evaluation of the metabolic to mitogenic potency ratio for B10-substituted insulin analogues. PLoS One. 2012;7, e29198.

22. Hansen BF, Glendorf T, Hegelund AC, Lundby A, Lutzen A, Slaaby R, et al. Molecular characterisation of long-acting insulin analogues in comparison with human insulin, IGF-1 and insulin X10. PLoS One. 2012;7, e34274.

23. Knudsen $L$, Hansen BF, Jensen $P$, Pedersen TA, Vestergaard $K$, Schaffer $L$, et al. Agonism and antagonism at the insulin receptor. PLoS One. 2012;7, e51972.

24. Liefvendahl E, Arnqvist HJ. Mitogenic effect of the insulin analogue glargine in malignant cells in comparison with insulin and IGF-I. Horm Metab Res. 2008;40:369-74.

25. Mayer D, Shukla A, Enzmann H. Proliferative effects of insulin analogues on mammary epithelial cells. Arch Physiol Biochem. 2008;114:38-44.

26. Milazzo G, Sciacca L, Papa V, Goldfine ID, Vigneri R. ASPB10 insulin induction of increased mitogenic responses and phenotypic changes in human breast epithelial cells: evidence for enhanced interactions with the insulin-like growth factor-I receptor. Mol Carcinog. 1997;18:19-25.

27. Oleksiewicz MB, Bonnesen C, Hegelund AC, Lundby A, Holm GM, Jensen $M B$, et al. Comparison of intracellular signalling by insulin and the hypermitogenic AspB10 analogue in MCF-7 breast adenocarcinoma cells. J Appl Toxicol. 2011;31:329-41.

28. Pierre-Eugene C, Pagesy P, Nguyen TT, Neuille M, Tschank G, Tennagels N, et al. Effect of insulin analogues on insulin/IGF1 hybrid receptors: increased activation by glargine but not by its metabolites M1 and M2. PLoS One. 2012;7, e41992.

29. Sciacca L, Cassarino MF, Genua M, Vigneri P, Giovanna Pennisi M, Malandrino P, et al. Biological Effects of Insulin and Its Analogs on Cancer Cells With Different Insulin Family Receptor Expression. J Cell Physiol. 2014:229:1817.

30. Shukla A, Enzmann H, Mayer D. Proliferative effect of Apidra (insulin glulisine), a rapid-acting insulin analogue on mammary epithelial cells. Arch Physiol Biochem. 2009;115:119-26.

31. Shukla A, Grisouard J, Ehemann V, Hermani A, Enzmann H, Mayer D. Analysis of signaling pathways related to cell proliferation stimulated by insulin analogs in human mammary epithelial cell lines. Endocr Relat Cancer. 2009;16:429-41.
32. Staiger $\mathrm{K}$, Hennige $\mathrm{AM}$, Staiger $\mathrm{H}$, Haering $\mathrm{HU}$, Kellerer M. Comparison of the mitogenic potency of regular human insulin and its analogue glargine in normal and transformed human breast epithelial cells. Horm Metab Res. 2007:39:65-7.

33. Teng JA, Hou RL, Li DL, Yang RP, Qin J. Glargine promotes proliferation of breast adenocarcinoma cell line MCF-7 via AKT activation. Horm Metab Res. 2011;43:519-23.

34. Ter Braak B, Siezen CL, Kannegieter N, Koedoot E, van de Water B, van der Laan JW. Classifying the adverse mitogenic mode of action of insulin analogues using a novel mechanism-based genetically engineered human breast cancer cell panel. Arch Toxicol. 2014;88:953.

35. Weinstein $D$, Simon M, Yehezkel $E$, Laron Z, Werner $H$. Insulin analogues display IGF-I-like mitogenic and anti-apoptotic activities in cultured cancer cells. Diabetes Metab Res Rev. 2009;25:41-9.

36. Gallagher EJ, Alikhani N, Tobin-Hess A, Cannata D, Tennagels N, Werner U, et al. The effect of rhIGF-1 and insulin analogue AspB10 on mammary tumour growth and progression in a mouse model of type 2 diabetes. Diabetologia. 2012;55:S289.

37. Stammberger I, Bube A, Durchfeld-Meyer B, Donaubauer H, Troschau G. Evaluation of the carcinogenic potential of insulin glargine (LANTUS) in rats and mice. Int J Toxicol. 2002;21:171-9.

38. Stammberger I, Essermeant L. Insulin glargine: A reevaluation of rodent carcinogenicity findings. Int J Toxicol. 2012;31:137-42.

39. Tennagels N, Welte S, Hofmann M, Brenk P, Schmidt R, Werner U. Differences in metabolic and mitogenic signalling of insulin glargine and insulin aspart B10 in rats. Diabetologia. 2013;56:1826-34.

40. ter Braak B, Siezen C, Speksnijder EN, Koedoot E, van Steeg H, Salvatori DC, et al. Mammary gland tumor promotion by chronic administration of IGF1 and the insulin analogue AspB10 in the p53R270H/(+)WAPCre mouse model. Breast Cancer Res. 2015;17:14.

41. Bodmer M, Meier C, Krahenbuhl S, Jick SS, Meier CR. Long-term metformin use is associated with decreased risk of breast cancer. Diabetes Care. 2010;33:1304-8.

42. Bordeleau L, Yakubovich N, Dagenais GR, Rosenstock J, Probstfield J, Yu PC, et al. The Association of Basal Insulin Glargine and/or n-3 Fatty Acids With Incident Cancers in Patients With Dysglycemia. Diabetes Care. 2014;37:1360-6.

43. Carstensen B, Witte DR, Friis S. Cancer occurrence in Danish diabetic patients: duration and insulin effects. Diabetologia. 2012;55:948-58

44. Chang CH, Toh S, Lin JW, Chen ST, Kuo CW, Chuang LM, et al. Cancer risk associated with insulin Glargine among adult type 2 diabetes patients - a nationwide Cohort study. PLoS ONE. 2011;6, e21368.

45. Cleveland RJ, North KE, Stevens J, Teitelbaum SL, Neugut Al, Gammon MD. The association of diabetes with breast cancer incidence and mortality in the Long Island Breast Cancer Study Project. Cancer Causes Control. 2012;23:1193-203.

46. Dejgaard A, Lynggaard H, Rastam J, Krogsgaard TM. No evidence of increased risk of malignancies in patients with diabetes treated with insulin detemir: A meta-analysis. Diabetologia. 2009;52:2507-12.

47. Fagot JP, Blotiere PO, Ricordeau P, Weill A, Alla F, Allemand H. Does insulin glargine increase the risk of cancer compared with other basal insulins? A French nationwide cohort study based on national administrative databases. Diabetes Care. 2013;36:294-301.

48. Ferrara A, Lewis JD, Quesenberry Jr CP, Peng T, Strom BL, Van Den Eeden SK, et al. Cohort study of pioglitazone and cancer incidence in patients with diabetes. Diabetes Care. 2011;34:923-9.

49. Grimaldi-Bensouda L, Cameron D, Marty M, Barnett AH, Penault-Llorca F, Pollak $M$, et al. Risk of breast cancer by individual insulin use: an international multicenter study. Diabetes Care. 2014;37:134-43.

50. Gu YJ, Wang CF, Zheng Y, Hou XH, Mo YF, Yu WH, et al. Cancer Incidence and Mortality in Patients with Type 2 Diabetes Treated with Human Insulin: A Cohort Study in Shanghai. Plos One. 2013;8, e53411.

51. Habel LA, Danforth KN, Quesenberry CP, Capra A, Van Den Eeden SK, Weiss NS, et al. Cohort study of insulin glargine and risk of breast, prostate, and colorectal cancer among patientswith diabetes. Diabetes Care. 2013;36:3953-60.

52. Home PD, Lagarenne P. Combined randomised controlled trial experience of malignancies in studies using insulin glargine. Diabetologia. 2009;52:2499-506. 
53. Hsieh MC, Lee TC, Cheng SM, Tu ST, Yen MH, Tseng CH. The influence of type 2 diabetes and glucose-lowering therapies on cancer risk in the Taiwanese. Exp Diabetes Res. 2012;2012:413782.

54. Koro C, Barrett S, Qizilbash N. Cancer risks in thiazolidinedione users compared to other anti-diabetic agents. Pharmacoepidemiol Drug Saf. 2007;16:485-92.

55. Kostev K. Risk of breast cancer in patients on long-acting insulin analogues in comparison with those on human insulin. Diabetologia. 2012;55:1554-5.

56. Lind $M$, Fahlen $M$, Eliasson $B$, Oden $A$. The relationship between the exposure time of insulin glargine and risk of breast and prostate cancer: An observational study of the time-dependent effects of antidiabetic treatments in patients with diabetes. Primary Care Diabetes. 2012;6:53-9.

57. Ljung R, Talback M, Haglund B, Jonasson JM, Gudbjornsdottir S, Steineck G. Insulin glargine use and short-term incidence of malignancies - a three-year population-based observation. Acta Oncol. 2011;50:685-93.

58. Mannucci E, Monami M, Balzi D, Cresci B, Pala L, Melani C, et al. Doses of insulin and its analogues and cancer occurrence in insulin-treated type 2 diabetic patients. Diabetes Care. 2010;33:1997-2003.

59. Morden NE, Liu SK, Smith J, Mackenzie TA, Skinner J, Korc M. Further exploration of the relationship between insulin glargine and incident cancer: a retrospective cohort study of older Medicare patients. Diabetes Care. 2011;34:1965-71.

60. Neumann A, Weill A, Ricordeau P, Fagot JP, Alla F, Allemand H. Pioglitazone and risk of bladder cancer among diabetic patients in France: a populationbased cohort study. Diabetologia. 2012;55:1953-62.

61. Onitilo AA, Stankowski RV, Berg RL, Engel JM, Glurich I, Williams GM, et al. Type 2 diabetes mellitus, glycemic control, and cancer risk. European J Cancer Prev. 2014;23:134-40.

62. Redaniel MT, Jeffreys M, May MT, Ben-Shlomo Y, Martin RM. Associations of type 2 diabetes and diabetes treatment with breast cancer risk and mortality: a population-based cohort study among British women. Cancer Causes Control. 2012;23:1785-95.

63. Rosenstock J, Fonseca V, McGill JB, Riddle M, Halle JP, Hramiak I, et al. Similar risk of malignancy with insulin glargine and neutral protamine Hagedorn (NPH) insulin in patients with type 2 diabetes: Findings from a 5 year randomised, open-label study. Diabetologia. 2009;52:1971-3.

64. Ruiter R, Visser LE, van Herk-Sukel MP, Coebergh JW, Haak HR, GeelhoedDuijvestijn $\mathrm{PH}$, et al. Risk of cancer in patients on insulin glargine and other insulin analogues in comparison with those on human insulin: results from a large population-based follow-up study. Diabetologia. 2012;55:51-62.

65. Sturmer T, Marquis MA, Zhou H, Meigs JB, Lim S, Blonde L, et al. Cancer incidence among those initiating insulin therapy with glargine versus human NPH insulin. Diabetes Care. 2013;36:3517-25.

66. Suissa S, Azoulay L, Dell'Aniello S, Evans M, Vora J, Pollak M. Long-term effects of insulin glargine on the risk of breast cancer. Diabetologia. 2011;54:2254-62.

67. Vallarino C, Perez A, Fusco G, Liang H, Bron M, Manne S, et al. Comparing pioglitazone to insulin with respect to cancer, cardiovascular and bone fracture endpoints, using propensity score weights. Clinical drug Invest. 2013;33:621-31.

68. Sommerfeld MR, Muller G, Tschank G, Seipke G, Habermann P, Kurrle R et al. In vitro metabolic and mitogenic signaling of insulin glargine and its metabolites. PLoS One. 2010;5, e9540.

69. Kuerzel GU, Shukla U, Scholtz HE, Pretorius SG, Wessels DH, Venter C, et al. Biotransformation of insulin glargine after subcutaneous injection in healthy subjects. Curr Med Res Opin. 2003;19:34-40.

70. Bolli GB, Hahn AD, Schmidt R, Eisenblaetter T, Dahmen R, Heise T, et al. Plasma exposure to insulin glargine and its metabolites M1 and M2 after subcutaneous injection of therapeutic and supratherapeutic doses of glargine in subjects with type 1 diabetes. Diabetes Care. 2012;35:2626-30.

71. Jorgensen L, Dideriksen L, Drejer K. Carcinogenic effect of the human insulin analog b10 asp in female rats. In: Diabetologia: 1992. 175 Fifth ave, New York, NY 10010: Springer Verlag; 1992. p. A3-3.

72. Rose DP, Vona-Davis L. The cellular and molecular mechanisms by which insulin influences breast cancer risk and progression. Endocr Relat Cancer. 2012;19:R225-241.

73. Van Oosterhout JP, Van der Laan JW, De Waal EJ, Olejniczak K, Hilgenfeld M, Schmidt $V$, et al. The utility of two rodent species in carcinogenic risk assessment of pharmaceuticals in Europe. Regul Toxicol Pharmacol. 1997:25:6-17.
74. Col NF, Ochs L, Springmann V, Aragaki AK, Chlebowski RT. Metformin and breast cancer risk: a meta-analysis and critical literature review. Breast Cancer Res Treat. 2012;135:639-46.

75. Gerstein HC, Bosch J, Dagenais GR, Diaz R, Jung H, Maggioni AP, et al. Basal insulin and cardiovascular and other outcomes in dysglycemia. N Engl J Med. 2012;367:319-28.

76. Besic N, Satej N. Insulin glargine versus other types of basal insulin-clinical and tumor characteristics in patients with breast carcinoma. BMC Res Notes. 2013;6:416.

77. Peairs KS, Barone BB, Snyder CF, Yeh HC, Stein KB, Derr RL, et al. Diabetes mellitus and breast cancer outcomes: a systematic review and metaanalysis. J Clin Oncol. 2011;29:40-6.

78. Luo J, Virnig B, Hendryx M, Wen S, Chelebowski R, Chen C, et al. Diabetes, diabetes treatment and breast cancer prognosis. Breast Cancer Res Treat. 2014:148:153-62

79. Hou G, Zhang S, Zhang X, Wang P, Hao X, Zhang J. Clinical pathological characteristics and prognostic analysis of 1,013 breast cancer patients with diabetes. Breast Cancer Res Treat. 2013;137:807-16.

80. Renehan AG, Yeh HC, Johnson JA, Wild SH, Gale EAM, Moller H, et al. Diabetes and cancer (2): evaluating the impact of diabetes on mortality in patients with cancer. Diabetologia. 2012;55:1619-32.

81. Lipscombe LL, Goodwin PJ, Zinman B, McLaughlin JR, Hux JE. The impact of diabetes on survival following breast cancer. Breast Cancer Res Treat. 2008:109:389-95

82. Currie CJ, Poole CD, Evans M, Peters JR, Morgan CL. Mortality and other important diabetes-related outcomes with insulin vs other antihyperglycemic therapies in type 2 diabetes. J Clin Endocrinol Metab. 2013;98:668-77.

83. loacara S, Guja C, lonescu-Tirgoviste C, Fica S, Roden M. Cancer specific mortality in insulin-treated type 2 diabetes patients. PLoS One. 2014;9:e93132

\section{Submit your next manuscript to BioMed Central and take full advantage of:}

- Convenient online submission

- Thorough peer review

- No space constraints or color figure charges

- Immediate publication on acceptance

- Inclusion in PubMed, CAS, Scopus and Google Scholar

- Research which is freely available for redistribution 